\title{
Dynamics of Submergence and Uplift of a Sedimentary Basin Underlain by a Phase-Change Boundary
}

\author{
Richard J. 0'Conneli ${ }^{1}$ and G. J. Wasserburg \\ Division of Geological and Planetary Sciences \\ California Institute of Technology, Pasadena, California 91109
}

A procedure is given for calculating the evolution of a sedimentary basin underlain by a phase-change boundary that is in isostatic equilibrium. The equation of motion of the water depth $w(t)$ as a function of the water depth and as a function of the sedimentation rate $d s / d t$ is

$$
Q_{w}(w) \frac{d w}{d t}+Q_{\cdot} \frac{d s}{d t}+\left(1-\rho_{1} / \rho_{2}\right) \frac{d l}{d t}=0
$$

where $l(t)$ is the lag of the phase boundary behind the transient equilibrium position. Numerically precise integrals of this equation are given for different time regimes by use of certain approximations for the temperature field that determine $d l / d t$. Solutions are demonstrated that are attenuated, explosive, or near periodic, depending on the physical parameters used. The results clearly show the possibility of depositing very thick sedimentary sections and the existence of self-sustained oscillations (above and below sea level) for a sedimentary basin of this type.

There is no doubt that phase changes are a significant feature of the earth's interior. Thus, an understanding of the theoretical consequences of the dynamical behavior of a phase-change boundary is of general interest. Considerable speculation has been made as to whether or not a phase change at the Mohorovicic boundary could be a significant cause of mountain building or of thick sedimentary sections. There is, however, considerable doubt [see Wetherill, 1961] that the $\mathrm{M}$ discontinuity is a phase change. Furthermore, the more recent discovery of plate tectonics makes doubtful any simple phase-change model of crustal structure. Understanding the basic characteristics of the dynamical behavior is nonetheless of considerable importance. The general problem is dependent on many physical parameters, and the field equations are nonlinear. Some previous investigations have been carried out in which heavy computer calculations were used (see Joyner [1967] for a correct calculation). In the present work, we shall outline and summarize the general one-dimensional analytic theory based on the work of $O^{\prime}$ Connell and Wasserburg [1967] and $O^{\prime}$ Connell [1969] (to be referred to as ' $O W$ ' and ' $O$,' respectively). These workers have shown how to obtain approximate solutions to the heat-transport problem for a moving phase boundary. In particular, $O$ gives a rather detailed development of the analytic results used in this paper, which permits the discussion of the

\footnotetext{
${ }^{1}$ Now at the Department of Geological Sciences and Center for Earth and Planetary Physics, Harvard University, Cambridge, Massachusetts 02138.
} 
geophysical problem without the direct encumberment of the field equations for heat flow. The detailed analysis of the various analytic approximations will not be given here. The reader is referred to $\mathrm{OW}$ and to $\mathrm{O}$ for particulars.

This paper extends the paper OW by presenting the phase-boundary dynamics in the framework of an isostatically compensating sedimentation basin, using the solutions presented in OW but generalized in a fundamental way to a physically identifiable problem. Certain characteristics of the geophysical problem are identified (e.g., stability) and the existence of different classes of solutions, such as oscillatory, are demonstrated. Much of this is contained in $\mathrm{O}$ but is here presented in a more expository form. We shall attempt in our presentation to outline the dissection of the problem into well-defined 'regimes' and to give the fundamental conclusions. It is our hope that other workers may be able to use this condensed presentation to simply analyze the geophysical consequences of any particular phase-change model.

The essential problem that we have attacked is the motion of a first-order phase boundary subjected to the pressure (transmitted instantaneously) of a layer of overlying sediments, assuming that some mechanism operates to isostatically compensate for the change in surface load and elevation that results. This will then constitute the evolution through time of a water-filled sedimentary basin that may finally be filled with sediments, is then uplifted and eroded, and may again subside. The equation of motion for the change of water depth $d w$ with the change in sediment thickness $d s$ as related to the position of the phase boundary $M$ will be given by a general equation of the form $d w(t) / d s(t)=\Phi(M(t))$. The integral of this equation will provide a description of the development of the sedimentary basin with time when the law of sedimentation (and erosion) $d s / d t$ is given and will yield the thickness of accumulated (or remaining) sediments and the height and duration of uplift that may result.

\section{MODEL FOR ANALYTIC DESCRIPTION}

The basic model is shown below and follows that used before (OW, pp. 396-397).

\begin{tabular}{|c|c|}
\hline Water & $\rho w$ \\
\hline Sediment & $\rho_{s}, K$, \\
\hline Low-density phase & $\rho_{1}, K_{1}$ \\
\hline High-density phase & $\rho_{2}, K_{2}$ \\
\hline Asthenosphere & $\rho_{0}$ \\
\hline
\end{tabular}

Sea level

$20 \equiv x_{s}-x_{w}$

Top of sediments

$s \equiv-x$

Bottom of sediments

Phase boundary

Thermal lower boundary

Level of isostatic compensation

The coordinate system is fixed with respect to the low-density phase, i.e., the region above the phase boundary $x=M$. The origin $x=0$ is at the top of the low-density phase, which is the interface between this phase and any sediments that may be present. Elevation is expressed relative to mean 
sea level $x_{w}$. The water depth in a basin is $w=x_{s}-x_{w}$, where $x_{8}$ is the top of the sediments. If the surface is above sea level, $w$ will be negative and will correspond to the elevation above sea level. The sediment thickness is $s=-x_{s}$. We shall assume that at time $t=0, s(0)=0$.

The level $x=b$ is fixed in the material of the high-density phase and is the level at which a thermal boundary condition is prescribed, so that the heattransfer equation associated with the moving phase boundary is solved in the region above $x=b$. Somewhere beneath this region is the asthenosphere, in which the material can flow to maintain isostatic equilibrium. This region is bounded below by the level $x_{c}$, which is fixed relative to the center of the earth and is thus fixed relative to $x_{w}$ so that $x_{c}-x_{w}$ is constant. The model is said to be in isostatic equilibrium when the mass per unit area in a vertical column above $x_{c}$ is constant. The case of no isostatic compensation can be treated by letting $\rho_{c} \rightarrow \infty$.

In this model the reference elevation $x_{w}$, which is assumed to be stable with respect to the center of the earth, coincides with the top of the water. That is, the basin is assumed to be connected with the ocean so that the water level in the basin coincides with the mean sea level. If this is not the case, e.g., if the basin became isolated or if one were considering an inland sea where the water level would not be maintained at mean sea level, the model would require modification. The modification can be made by reconsidering the mass balance in the vertical column with these considerations explicitly included.

In order to treat the motion of the phase boundary $M(t)$, we must construct a thermal model of the crust and upper mantle. For our purpose a relatively simple model is sufficient; the model is directly related, however, to a realistic picture of the thermal state of the crust and upper mantle, and the important parameters and features of the model are obtained from consideration of the actual geophysical situation. The model we use is a one-dimensional layered region that is finite. At the surface (the top of the sediments) the temperature is taken as constant in time. At the bottom of the region another boundary condition must be fixed. In this paper, we shall assume that the heat flux is constant at a level $x=b$, which is at some depth in the earth. The effect of this lower boundary condition has been investigated in detail in $\mathrm{OW}$, where constant temperature and constant flux cases were compared. The important parameters are the initial depth of the phase boundary $M(0)$, the slope of the Clapeyron curve $d T_{c} / d P \equiv G$, and the initial temperature distribution near the phase boundary.

In our model, we shall neglect the presence of radioactive heat sources in the dynamic equations. This has been previously justified (OW, pp. 347, 384390). In terms of our present model, this approximation means that the gradient of the initial temperature distribution in the region through which the phase boundary moves is taken as constant, with a value

$$
\left.\frac{\partial T(x, 0)}{\partial x}\right|_{x \approx M}=\frac{J_{M}}{K_{1}}
$$

where $J_{M}$ is the heat flux at the phase boundary before the onset of any dynamic 
effects, and $K_{1}$ is the thermal conductivity of the low-density phase that was originally present in the region through which the phase boundary moves. To take into account the thermal blanketing effect of the sediments deposited, we must know the heat flow into the base of the sedimentary column. We shall take this flux $J_{s}$ to be the initial value at $x=0$. The temperature in the superimposed sediments will then be proportional to $J_{g} K_{8}$ where $K_{s}$ is the conductivity of sediments. In general, $J_{s}>J_{M}$, owing to the presence of radioactive heat sources in the upper crust. Both $J_{M} / K_{1}$ and $J_{s} / K_{8}$ may be regarded as mean effective temperature gradients in the respective regions and should correspond to values taken from a realistic geothermal model (cf. Figure 1). All these parameters are obtained from consideration of the supposed actual state of the crust and upper mantle. Note that although heat sources may be neglected in the solution of the dynamical problem, their effect in determining the initial steady-state temperature distribution must still be taken into account. The solution for the temperature field obtained from the dynamical solution corresponds to the change of the temperature distribution from the initial steady state (cf. OW).

If there were no thermal transients associated with the movement of the phase boundary and the deposition of sediments on the surface, the phase boundary would be at the intersection of the Clapeyron curve and the initial temperature distribution $M_{e}(t)$, as shown in Figure 1. Because of the latent heat of the phase change, there will be a thermal transient owing to the release of latent heat at the phase boundary, and the phase boundary $M(t)$ will actually lag a distance $l(t)$ behind $M_{e}(t)$. Since we can compute $M_{e}(t)$ at any time from a knowledge of the pressure and the initial state of the model, we can formally write $M(t)=M_{\theta}(t)+l(t)$, in which case all the information about the dynamic response of the phase boundary is contained in the lag $l(t)$, which must be obtained from a detailed study of the heat-transport problem. For our model, neglecting heat sources,

$$
M(0)-M_{e}(t)=\frac{G\left[P_{0}(t)-P_{0}(0)\right]}{\left[G \rho_{1} g-J_{M} / K_{1}\right]}
$$

where the Clapeyron slope is $d T_{c} / d P=G, g$ is the acceleration of gravity and $P_{0}(t)$ is the pressure at the surface $x=0$. Correspondingly, $l(t) \equiv M(t)-$ $M_{e}(t)=M(0)-M_{e}(t)-[M(0)-M(t)]$.

$M(0)-M_{e}(t)$ is thus obtained by considering the intersection of the Clapeyron and temperature curves for a realistic model; this is obtained by simple geometrical considerations. If heat sources are included in the model, the equation for $M(0)-M_{e}(t)$ will be quadratic; nevertheless, if $J_{M}$ is regarded as the average flux between $M(0)$ and $M_{\theta}(t)$, a linear relation can be obtained. In so far as the concentration of heat sources is small, $J_{M r}$ can be taken as the heat flux at $M(0)$ with negligible error. All the information, including effects of thermal blanketing, about the moving phase boundary is thus associated with the lag $l(t)$. This involves the solution of a nonlinear diffusion equation with a moving boundary, which has presented mathematical difficulties in previous 
Fig. 1. (a) Geotherm and Clapeyron curve for supposed crust and upper mantle. The initial position of the phase boundary $M(0)$ is at the intersection of the two curves. (b) Detail showing intersection of initial geotherm and Clapeyron curve at two different times. The displacement $M(0)-$ $M_{\theta}(t)$ is directly calculable from the parameters specifying the geotherm and Clapeyron curve. (c) Relation between geotherm and Clapeyron curve implied by model used to calculate transient thermal effects due to the moving phase boundary. In so far as the curvature of $T(x, 0)$ is small between $M(0)$ and $M_{\theta}(t)$, the replacement of the quadratic temperature distribution with a linear one introduces no significant error. $J_{U}$ is the average heat flux between $M(0)$ and $M_{\theta}(t)$, so that $J_{x x} / K_{1}$ is the average temperature gradient. (d) Thermal state of crust and upper mantle implied by our model used to solve moving phase boundary problem. Portions of the geotherm shown as solid lines are assumed to be linear. In addition, a thermal boundary condition is specified at depth $b$. The model still corresponds closely to the supposed actual crust and upper mantle, shown in (a). (e) Temperature field near moving phase boundary. $M_{\theta}(t)$ is defined as the intersection of the Clapeyron curve $T_{c}(x, t)$ and the initial temperature curve $T(x, 0)$. The phase boundary $M(t)$ will lag a distance $l(t)$ behind $M_{e}(t)$ because of transient thermal effects due to the release of the latent heat of the phase change. The thermal blanketing effect of sediments on the surface is also indicated.

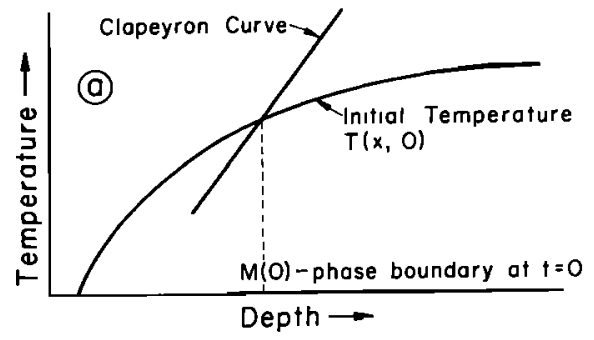

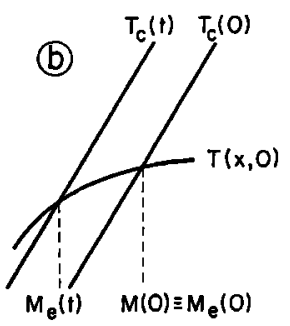

Actual model

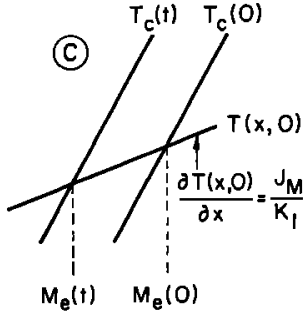

Implied model
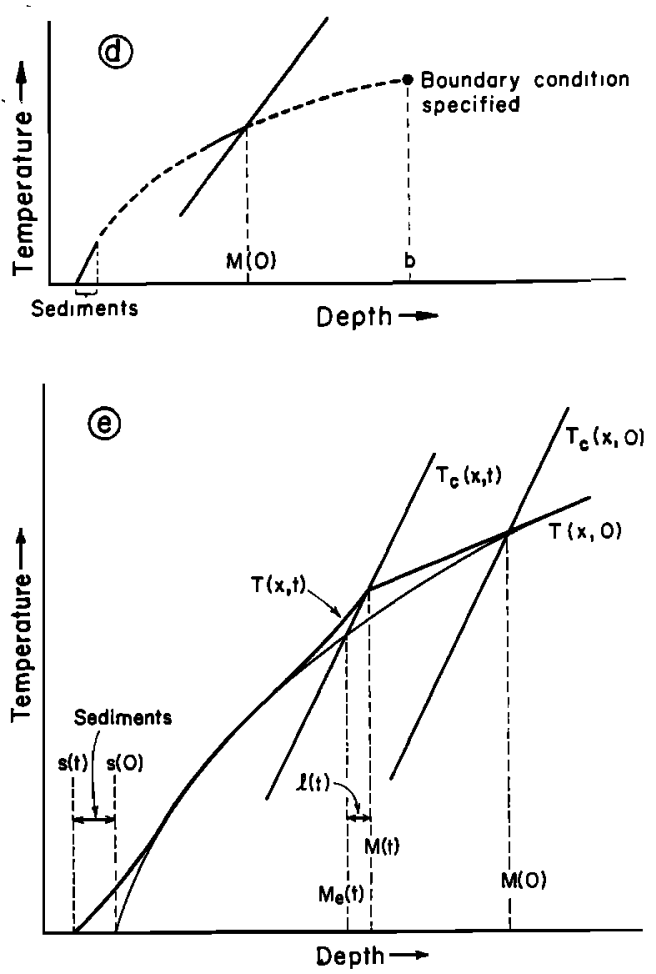
studies of this problem. The solution of this problem has been obtained by OW and will be used to calculate $l(t)$ in this paper.

We thus obtain the following basic relation for an isostatic model between water depth $w$, initial water depth $w_{0}$, sediment thickness $s$, (with $s(0)=0$ ), and the lag of the phase boundary $l$, (equation 67 of $\mathrm{OW}$ )

$$
Q_{w} w-Q_{w_{0}} w_{0}+Q_{o} s=-l_{\rho_{1}}\left(\frac{1}{\rho_{1}}-\frac{1}{\rho_{2}}\right)
$$

Here $Q_{i}$ are defined by the initial conditions and the sign of $w$

$$
Q_{i} \equiv \rho_{i}\left[\frac{1}{\rho_{i}}-\frac{1}{\rho_{c}}-\frac{1}{W}\left(\frac{1}{\rho_{1}}-\frac{1}{\rho_{2}}\right)\right] \quad i=s, w, w_{0}
$$

and

$$
W \equiv 1-\frac{J_{M}}{K_{1} G_{\rho_{1} g} g} \equiv 1-\left.\frac{\partial T(x, 0)}{\partial x}\right|_{x-M-} /\left.\frac{d T_{c}(x)}{d x}\right|_{x-M}
$$

The parameter $\rho_{w}$ represents the water density if $w>0\left(\rho_{w}=1\right)$; if the sediment surface $x_{s}(t)$ is above sea level $(w<0)$, then $\rho_{w}=0$. The critical quantities from the initial temperature depth curve are the initial position $M(0)$ and temperature $T_{e}(M(0))$ of the phase boundary and the temperature gradient at this point. The term $G_{\rho_{1}} g$ is the spatial gradient of the Clapeyron curve.

The parameter $W$ is the normalized difference between the Clapeyron slope and the temperature gradient, and ranges from 0 (equal slope) to 1 (infinite Clapeyron or zero temperature gradient). When $W$ is small, the angle of intersection of the two curves is also small, so that a small displacement of either curve can cause a large displacement of their intersection.

Important consequences of the dynamical motion may be seen by taking the derivative of equation 1 with respect to $s$ (equation 68 of $\mathrm{OW}$ )

$$
\frac{d w}{d s}=-\frac{Q_{s}}{Q_{w}}-\frac{\rho_{1}}{Q_{w}}\left(\frac{1}{\rho_{1}}-\frac{1}{\rho_{2}}\right) \frac{d l}{d s}
$$

The expression $d w / d s$ is the change in water depth with the change in sediment thickness. When this term is negative, the water depth decreases as sediments are deposited, and the basin is filling up. When the term is positive, the water depth increases as sediments are deposited, and the water basin becomes deeper. For $Q_{s}<0$ and $Q_{w}>0$, and if $l(t)=0$ (i.e., if the phase boundary responds instantaneously), then $d w / d s>0$. Such a case is defined as unstable. In general the lag will be a function of time, and the term in $d l / d s$ in (2) may cause $d w / d s$ to be $<0$, even for an unstable model; such a case is termed dynamically stable. A model may exhibit both dynamic instability and dynamic stability at different periods in its history. The development in time of a stable and an unstable basin is shown in Figure 2 . The unstable model is initially dynamically stable $(d w / d s$ $<0)$ but becomes dynamically unstable $(d w / d s>0)$ for a period before becorning dynamically stable again.

The cause of this type of instability can be understood by recalling that an isostatically compensated column can be regarded as a crustal block of mean 
density $\langle\rho\rangle$ floating in a fluid asthenosphere of density $\left.\rho_{o}\right\rangle\langle\rho\rangle$. The elevation of the surface is thus determined by Archimedes' principle. Ordinarily, with no phase changes present in the block, the mean density $\langle\rho\rangle$ changes little with the addition or removal of material to the block. If a phase change is present, however, the mean density $\langle\rho\rangle$ can change significantly if enough material changes state from one phase to another of different density. This can thus markedly affect the level

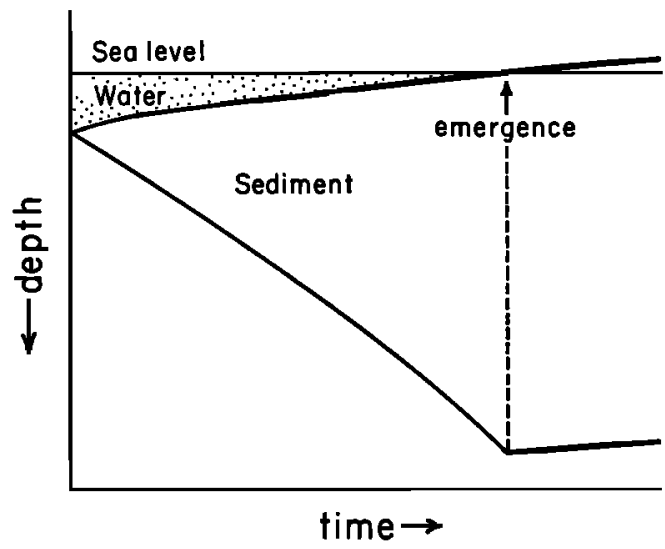

\author{
Stable: $Q_{s}>0$ \\ water depth \\ decreases with \\ sedimentotion
}

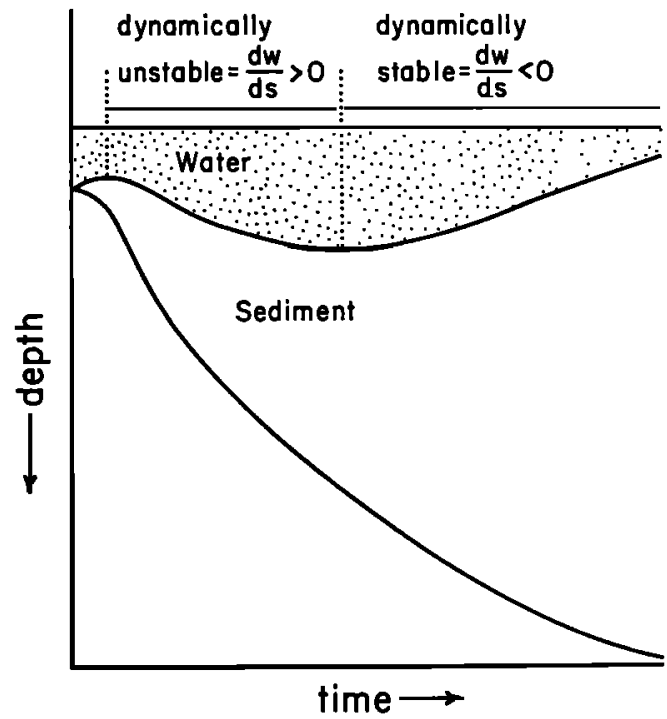

Unstable: $Q_{5}<0$

water depth can

increase with

sedimentation

Fig. 2. Comparison of stable and unstable behavior. For a stable model, the water depth always decreases as sediments are deposited, and the basin fills up. For an unstable model, there may be times of dynamic instability when the water depth increases as sediments are deposited. Thermal blanketing eventually causes the behavior to become dynamically stable again, so that the basin does eventually fill up. The time when the basin fills (water depth $=0$ ) is defined as $t_{t}$. 
to which the block sinks in the fluid, and hence the elevation of the top of the block above the surface. When material is added to a block with a phase change, the increase in pressure causes low-density material to change to high-density material, raising the mean density of the block and thus causing it to sink deeper. If enough material changes state, the mean density of the block can increase to the extent that the block sinks deep enough to lower the elevation of the surface. This is just the type of behavior defined above as unstable.

Thus instability can result from the phase boundary being very responsive to changes in pressure. An unstable model is characterized by a negative value of $Q_{B}$, which results when $W$ in equation $1 c$ is sufficiently small. This will occur if the ratio $J_{M} / K_{1} G_{\rho_{1}} g$ is near unity, i.e., if the slopes of the Clapeyron curve and temperature curve are nearly the same at the phase boundary. Then small shifts of the Clapeyron curve due to a pressure load at the surface can shift the intersection of the two curves a relatively large distance.

\section{FILLING OF A BASIN}

Before explicitly considering solutions giving the response of the phase boundary (i.e., $l(t)$ ) we can determine important characteristics of the model from more simple considerations. We consider a basin of initial water depth $w_{0}$ being filled by sediments.

The depth of the basin as a function of time is obtained by directly substituting the values of $l(t)$ and $s(t)$ into equation 1 and calculating $w(t)$. The sediment thickness $s(t)$ is determined by the law of deposition, and for a constant deposition rate $k$ is $s(t)=k t$. The basin depth $w(t)$ continuously changes with time until, at a time $t_{f}$, the basin is completely filled with sediments. The base of the column of sediments subsides as the basin fills and eventually accommodates a thickness $s\left(t_{f}\right)$ of sediments at a time $t_{f}$. At the time $t_{f}$ the surface of the sediments is just at sea level. This time $t_{f}$ may be found by evaluating equation 1 , and finding when $w(t)$, which is initially positive, passes through zero. The formal definition of $t_{j}$ is

$$
s\left(t_{f}\right)=\left[Q_{w_{0}} w_{\mathrm{u}}-l\left(t_{s}\right)\left(1-\frac{\rho_{1}}{\rho_{2}}\right)\right] / Q_{s}
$$

A simple limit for the total sediment thickness can be obtained by noting that $s\left(t_{f}\right)$ calculated from 3 is less than the maximum possible thickness, which would be attained if $l(t) \equiv 0$, i.e.,

$$
s_{\max }=\left(Q_{w_{0}} / Q_{s}\right) w_{0}
$$

The time at $s_{\max }$ is $s_{\max } /\langle d s / d t\rangle$ which is always greater than $t_{f}$, the time when the basin actually fills. $\langle d s / d t\rangle$ is the average sedimentation rate.

If there were no erosion after $t_{f}$, then the maximum uplift would occur. When $t \rightarrow \infty$, we obtain $l(t) \rightarrow s\left(t_{f}\right) J_{s} / K_{s} G_{\rho_{1}} g W$ (O'Connell [1969]; this result follows from the final steady-state temperature distribution, where the average heat flux through the sediments is $J_{8}$ ). The maximum elevation is

$$
-w=\left[Q_{s} s\left(t_{f}\right)-Q_{w_{0}} w_{0}+l(\infty)\left(1-\frac{\rho_{1}}{\rho_{2}}\right)\right] / Q_{w}
$$

where $Q_{w}$ is (as pointed out above) evaluated with $\rho_{w}=0$. 
It should be noted that, for an unstable model, $Q_{s}<0$, and (4) gives a meaningless result. For such cases, a static analysis with $l=0$ does not suffice, and the time dependence of the lag $l(t)$ is needed.

\section{UPLIFT AND EROSION}

So far in this discussion, we have started with a basin at $t=0$ and followed it until the time when the surface emerges. We also have found the maximum elevation by assuming no erosion.

Once the surface of the sediments is raised above sea level, erosional processes may take place $(d s(t) / d t<0)$.

Equation 1 is always valid and will obtain during uplift and erosion. In the region of uplift, however, the value of $Q_{w}$ is different from the value during sedimentation ( $\rho_{w}$ is zero during uplift). The differential version of this equation is then

$$
Q_{w}(w) \frac{d w}{d t}+Q_{0} \frac{d s}{d t}=-\frac{d l}{d t}\left(1-\frac{\rho_{1}}{\rho_{2}}\right)
$$

where this must be integrated from time $t_{f}$ (at which $s=s\left(t_{f}\right), l=l\left(t_{f}\right), w=0$ ), when the surface emerges, and one must use the value of $Q_{w}$ (with $\rho_{w}=0$ ) appropriate to the period of time during which the surface is above sea level. The evolution of the surface $w$ is determined by the erosion rate $d s / d t$, and the lag $d l / d t$, which in turn depends on the erosion rate and the past history of sedimentation.

In the differential form of equation 5 one can see the inherent nonlinearity, in that $Q_{w}$ is a function of $w$ (when $w$ changes sign, $Q_{w}$ assumes a different value). Nevertheless, equation 5 may still be directly integrated by considering the two separate intervals $0 \leq t \leq t_{f}$ and $t_{f} \leq t$ and matching the solutions for the two intervals at $t=t_{f}$. Since in each interval $Q_{w}$ will be constant, equation 5 will not be inherently nonlinear within the interval.

If erosion reduces the surface to sea level again, then another interval must be started, during which a new value of $Q_{w}$ will obtain. The solution will also have to be matched at the time of transition, so that it is continuous.

\section{SOLUTIONS FOR THE MOTION OF THE PHASE BOUNDARY}

The quantitative solution of the history of a model basin can be obtained from equation 1, once we know (1) the initial state of the system (the initial water depth $w_{0}$, etc.) ; (2) the law of sedimentation $[d s / d t=f(w, t, \ldots)]$; and (3) the position of the phase boundary $M(t)$ or, more conveniently, the lag $l(t)$. It is this last requirement that has presented mathematical difficulties that have previously limited the investigation of this problem.

The problem of the dynamical motion of the phase boundary has been studied in detail by OW and by O'Connell [1969]. The results of these investigations permit us to accurately describe the response of the phase boundary in very simple terms. The motion can be broken down into three different regimes of motion, as illustrated in Figure 3.

1. Short-term motion: the thermal effects due to the moving phase boundary are localized in the neighborhood of the boundary. In addition, the thermal 


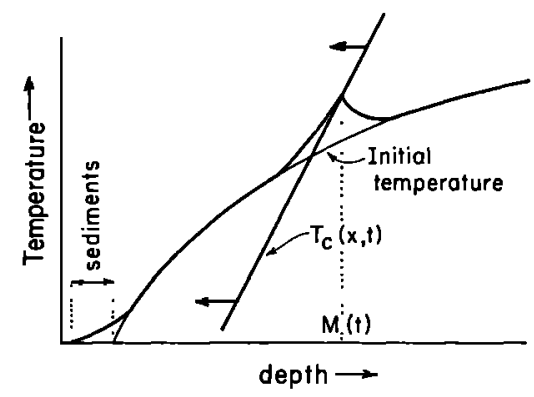

Short time behavior

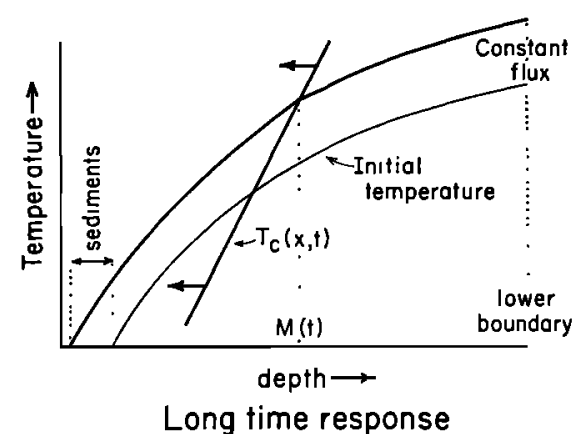

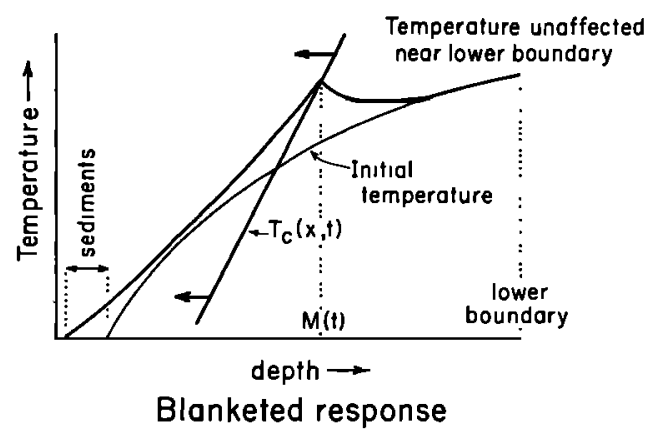

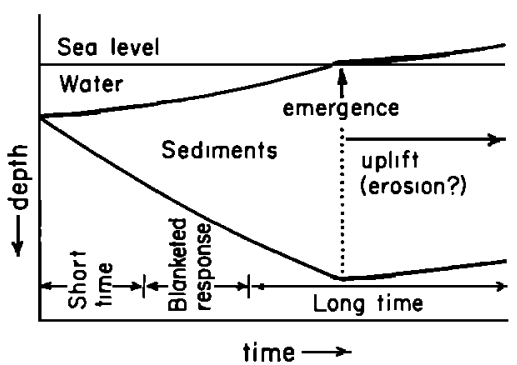

Fig. 3. Temperature field for different regimes of behavior. During short-time behavior, the temperature near the phase boundary $M(t)$ is not influenced either by boundary or by the sediments on the surface. During blanketed response, the temperature field near $M(t)$ is noticeably influenced by the thermal blanketing of the sediments. During long-time response, the temperature field in the whole region between the surface and the lower boundary is primarily influenced by the boundary conditions at the boundaries. Also shown is a representative illustration of the history of a sedimentary basin subsiding as it is filled with sediments. The behavior in the region where the sediments have emerged above sea level is shown for the special case of no erosion. (Note: Temperature effects exaggerated.)

blanketing effects of the sediments have not propagated to the neighborhood of the phase boundary.

2. Blanketed response: thermal blanketing of the sediments substantially interferes with the motion of the phase boundary.

3. Long-term response: there is no localization of any of the thermal effects; the temperature field is dominated by the conditions at the boundaries $x=x_{8}(t)$ and $x=b(t)$.

A typical time sequence is also shown in Figure 3. The time of filling of the basin for a given model may lie in any of the above regimes, depending on the initial water depth and the rate of sedimentation.

\section{EQUATIONS FOR SHORT-TERM BEHAVIOR}

In this regime the motion of the phase boundary is described by

$$
\frac{d}{d\left(\kappa_{1} t\right)^{1 / 2}}[M(0)-M(t)]=-\mu[M(0)-M(t)]+\frac{\mu}{W} \frac{\Delta P_{0}(t)}{\rho_{1}}
$$


where $\mu$ is a constant defined by

$$
\begin{aligned}
\mu & \equiv \frac{2}{\sqrt{\pi}} \frac{G \rho_{1} g W c_{1}}{L}\left[1+\left(\alpha^{\prime}\right)^{1 / 2}\right] \\
\alpha^{\prime} & \equiv\left(K_{2} \rho_{2} c_{1} / K_{1} \rho_{1} c_{2}\right)
\end{aligned}
$$

and $\Delta P_{0}(t)$ is the pressure on the surface $x=0$ due to sedimentation. $c i$ is the heat capacity, $L$ is the latent heat of the phase transition, and $\kappa_{1}$ is the thermal diffusivity. This is a generalization (including convective motion [ $O^{\prime} \mathrm{Con}$ nell, 1969, Appendix 1]) of equation 61 in our previous paper (OW, p. 391). The notation here is in terms of real dimensional parameters, rather than the dimensionless parameters in OW. The integral of 6 can be directly obtained for an arbitrary law of sedimentation

$$
M(0)-M(t)=\frac{\mu}{W} \rho_{1} e^{-\mu\left(\kappa_{1} t\right)^{1 / 2}} \int_{0}^{\left(\kappa_{2} t\right)^{1 / 9}} e^{\mu\left(\kappa_{1 z}\right)^{1 / 2}} \Delta P_{0}(z) d\left(\kappa_{1} z\right)^{1 / 2}
$$

We can obtain the lag $l(t)$

$$
\begin{aligned}
& l(t)=M(0)-M(t)-\left[M(0)-M_{\imath}(t)\right] \\
& l(t)=M(0)-M(t)-\frac{G \Delta P_{0}(t)}{\left[G \rho_{1} g-J_{M} / K_{1}\right]}
\end{aligned}
$$

With $\Delta P_{0}(t)=\rho_{s} g s(t)$ (neglecting any change in water depth), where $\rho_{s}$ is the sediment density, we thus obtain an expression for $l(t)$ in terms of the sediment thickness.

For the case of a constant sedimentation rate $d s / d t=k$, this gives

$$
l(t)=\frac{2}{W} \frac{\rho_{8}}{\rho_{1}} \frac{k}{\kappa_{1} \mu^{2}}\left[\mu\left(\kappa_{1} t\right)^{1 / 2}-1+e^{-\mu\left(\kappa_{2} t\right) 1 / 2}\right]
$$

This is always positive and therefore, from consideration of equation 2, cannot lead to dynamical instability. When a model is unstable, this term can compensate for $-Q_{s} / Q_{w}$ and cause it to be dynamically stable for this case. Since

$$
d l / d s=\frac{d l}{d t} / \frac{d s}{d t}
$$

decreases with time, it follows that an unstable system will start with dynamically stable motion, and the basin will begin to fill up. As $d l / d s$ decreases, the motion will become dynamically unstable when $d w / d s=0$ at a time satisfying

$$
\mu\left(\kappa_{1} t\right)^{1 / 2} \geq \frac{\rho_{s}}{W}\left(\frac{1}{\rho_{1}}-\frac{1}{\rho_{2}}\right) \frac{1}{\left(-Q_{s}\right)}
$$

Remember that $Q_{s}$ is negative for an unstable model. The value of this time will indicate whether dynamic instability will occur during the period when the short-time approximation is valid

\section{END OF SHORT-TERM BEHAVIOR AND ONSET OF LONG-TERM BEHAVIOR}

Short-term response will end when $t$ is greater than either $(M+s)^{2} / \kappa_{1} \pi$ or $(b-M)^{2} / \kappa_{2} \pi$, i.e., when the thermal effect from either the surface or the lower 
thermal boundary is noticeable near the phase boundary. Long-term behavior will apply when the effect of both boundaries is apparent at the phase boundary, i.e., when $t$ is greater than both $(M+s)^{2} / \kappa_{1} \pi$ and $(b-M)^{2} / \kappa_{2} \pi$.

In general, there will be a time interval between the end of short-term behavior and the start of long-term behavior during which neither type of behavior should strictly obtain. Usually, this period will be relatively short, so that errors introduced by using an inapplicable solution will be correspondingly small. The limits above are rather strict in that use of either short-term or long-term solutions beyond these limits has not resulted in significant errors for the wide variety of numerical models studied by us (OW, p. 361;0).

\section{LONG-TERM RESPONSE}

During this period the motion of the phase boundary is limited by the boundary conditions governing the flow of heat at the boundaries $x=0$ and $x=b$. To treat this, we use a quasi-steady-state approximation (QSSA) that represents the temperature field at each time by that steady-state solution appropriate to the instantaneous position of the phase boundary. The derivation and justification of this have been treated in OW, and more explicitly for this model, in 0 . The differential equation governing the motion of the phase boundary in this time domain is

where

$$
\frac{d}{d\left(\kappa_{1} t\right)}[M(0)-M(t)]=-\omega[M(0)-M(t)]+\varphi s(t)+\epsilon \frac{d s}{d t}
$$

$$
\begin{gathered}
\omega \equiv \frac{G \rho_{1} g W c_{1}}{H d} \\
\varphi \equiv \frac{G \rho_{s} g c_{1}}{H d} \\
\epsilon \equiv \frac{G \rho_{s} g c_{1}}{H \kappa_{1}}\left(\frac{d}{2}+\frac{c_{2}}{c_{1}} d^{\prime}\right) \\
H \equiv I+\frac{1}{2} c_{1} G \rho_{1} g W d+c_{2} d^{\prime}\left(G \rho_{1} g-\frac{\rho_{1} J_{M}}{\rho_{2} K_{2}}\right)
\end{gathered}
$$

and $d$ and $d^{\prime}$ are constants: $d$ is the average value of $M(t)+s(t)$, and $d^{\prime}$ is the average value of $b-M(t)$. The sediment thickness $s(t)$ is an arbitrary function of time.

This may be directly integrated to yield

$$
M(t)-M(t)=e^{-\omega \varepsilon_{1} t} \int_{\kappa_{1} \ell_{0}}^{\kappa_{1} t} e^{\omega \kappa_{1} t}\left[\varphi s(z)+\epsilon \frac{d s(z)}{d z}\right] d\left(\kappa_{1} z\right)
$$

Note that the independent variable is $\kappa_{1} t$ for this case, and not $\left(\kappa_{1} t\right)^{1 / 2}$, as it was for the short-term case. This fundamental difference reflects the difference in the nature of the flow of heat away from the moving phase boundary in the different regimes.

The solution for long-term behavior should be matched to that for shortterm behavior at the time (say, $\left.t_{0}\right)$ at which the transition is made. Thus $M\left(t_{0}\right)$ 
$-M(0)$ comes from the short-term solution and $M(t)-M\left(t_{0}\right)$ from the longterm solution, to give $M(t)-M(0)$.

By the time of long-term response, thermal blanketing is usually important. Therefore we shall defer the presentation of explicit solutions of the lag $l(t)$ for this regime until this effect has been included.

\section{THERMAL BLANKETING}

The influence of the surface on the motion of the phase boundary will certainly take place at a time

$$
\kappa_{1} t \geq \frac{[M(t)+s(t)]^{2}}{\pi}
$$

As the motion of the phase boundary depends on the heat transport away from it, a more precise criterion for the time by which thermal blanketing will dominate is (cf. OW, p. 396; O) the root of

$$
\kappa_{1} t=\frac{L K_{1} \rho_{s} d}{c_{1} J_{M} \rho_{1} W}+\frac{d^{2}}{6}\left[1+\frac{12}{\pi^{2}} \sum_{n=1}^{\infty} \frac{(-1)^{n}}{n^{2}} \exp \left(-\frac{n^{2} \pi^{2} \kappa_{1} t}{d^{2}}\right)\right]
$$

where $d \approx M(t)+s(t) \approx M(0)$ as before. Note that this criterion is independent of the sedimentation rate.

The effect of thermal blanketing is included in the solution for the moving phase boundary by adding a term to the differential equation (6 or 9) that corresponds to the change in the heat flux away from the phase boundary due to the presence of the thermal blanket of sediments (see $O$, Appendix 4, and appendix of this paper). This can be expressed in terms of a function $\Gamma(t)$. The equation for blanketed response in the regime of short-term motion is obtained by adding

$$
\left(2 c_{1} / L\right)\left(\kappa_{1} t\right)^{1 / 2} \Gamma(t)
$$

to the right-hand side of equation 6 . For long-term blanketed response, one adds

$$
\left(c_{1} / H\right) \Gamma(t)
$$

to the right-hand side of equation 9 . In either case, the resulting differential equation is still directly integrable.

For an arbitrary sedimentation function, we obtain from Duhamel's theorem [Carslaw and Jaeger, 1959, p. 30; 0]

$$
\Gamma(t)=\frac{2 \pi^{2} \kappa_{1}}{d^{3}} \sum_{n=1}^{\infty}(-1)^{n} n^{2} \exp \left(-\frac{\kappa_{1} n^{2} \pi^{2} t}{d^{2}}\right) \int_{0}^{l} \exp \left(\frac{\kappa_{1} n^{2} \pi^{2} z}{d^{2}}\right) \frac{J_{s}}{K_{s}} s(z) d z
$$

For a constant sedimentation rate, $s=k t$, we can obtain a more accurate expression that takes better into account the movement of the phase boundary and sedimentation surface relative to the coordinate system (appendix)

$$
\begin{aligned}
\Gamma(t)=-\frac{J_{s} k}{K_{s} \kappa_{1}} e^{d k / \kappa_{1}} & \left\{\frac{(1 / 2) k t+\kappa_{1} / k}{\sinh (1 / 2)\left(d k / 2 \kappa_{1}\right)}-d \frac{\cosh (1 / 2)\left(d k / 2 \kappa_{1}\right)}{\sinh ^{2}(1 / 2)\left(d k / 2 \kappa_{1}\right)}\right. \\
- & \left.\frac{2 \pi^{2}}{d^{3}} \sum_{n=1}^{\infty} \frac{(-1)^{n} n^{2}}{\left[\pi^{2} n^{2} / d^{2}+k^{2} / 4{\kappa_{1}}^{2}\right]^{2}} \exp \left[-\left(\frac{n^{2} \pi^{2}}{d^{2}}+\frac{k^{2}}{4 \kappa_{1}{ }^{2}}\right) \kappa_{1} t\right]\right\}
\end{aligned}
$$


For $(1 / 2)\left(d k / \kappa_{1}\right) \lessgtr 0.1$ this becomes

$$
\Gamma(t)=-\frac{J_{8} k}{K_{8} \kappa_{1}}\left[\frac{\kappa_{1} t}{d}-\frac{d}{6}-\frac{2 d}{\pi^{2}} \sum_{n=1}^{\infty} \frac{(-1)^{n}}{n^{2}} \exp \left(-\frac{\pi^{2} n^{2}}{d^{2}} \kappa_{1} t\right)\right]
$$

This equation can also be obtained from equation 12 .

\section{BLANKETED RESPONSE}

The solution for the lag of the phase boundary during the period of shortterm blanketed response can be found by integrating equation 6 with the appropriate expression for $\Gamma(t)$ included on the right-hand side. The quadrature is straightforward, although cumbersome. Since short-term response usually ends by the time thermal blanketing becomes important, this solution is of limited utility and will not be presented here. It may be found in $O$ (p. 116) for a constant sedimentation rate.

The most useful solution is that for long-term blanketed response, since any model must ultimately enter this regime. The solution for a constant sedimentation rate $\dot{s}=k$ is obtained from equations 9 and 13 or 14

$$
\begin{array}{r}
l(t)=k\left(\frac{\varphi}{\kappa_{1} \omega^{2}}-\frac{\epsilon}{\omega}\right)\left(1-e^{-\omega \kappa_{1} t}\right)+\frac{a_{1} d}{\omega}\left(\omega \kappa_{1} t-1+e^{-\omega \kappa_{1} t}\right)+a_{2} d\left(1-e^{-\omega \kappa_{1} t}\right) \\
-d \omega a_{3} \sum_{n=1}^{\infty} \frac{h_{n}}{\left(\omega-\nu_{n}\right)}\left(e^{-\eta_{n} \kappa_{1} t}-e^{-\omega \kappa_{1} t}\right)
\end{array}
$$

where

$$
\begin{aligned}
& \nu_{n} \equiv \frac{n^{2} \pi^{2}}{d^{2}}+\frac{k^{2}}{4 \kappa_{1}^{2}} \\
& a_{1} \equiv \frac{J_{s} k^{2} \exp \left(d k / 2 \kappa_{1}\right)}{2 K_{s} \kappa_{1}{ }^{2} \sinh \left(d k / 2 \kappa_{1}\right) G \rho_{1} g W} \\
& a_{2} \equiv \frac{J_{s} k \exp \left(d k / 2 \kappa_{1}\right)}{2 K_{s} \kappa_{1} \sinh \left(d k / 2 \kappa_{1}\right) G \rho_{1} g W}\left[\frac{2 \kappa_{1}}{k}-\frac{d}{\tanh \frac{d}{\left(d k / 2 \kappa_{1}\right)}}\right] \\
& a_{3}=\frac{2 J_{s} k \pi^{2} \exp \left(d k / 2 \kappa_{1}\right)}{K_{s} \kappa_{1} d^{3} G \rho_{1} g W} \\
& h_{n} \equiv \frac{(-1)^{n} n^{2}}{\left(\frac{n^{2} \pi^{2}}{d^{2}}+\frac{k^{2}}{4 \kappa_{1}{ }^{2}}\right)^{2}}
\end{aligned}
$$

When $\left(d k / 2 \kappa_{1}\right)^{2} \ll 1$, the above constants become

$$
\begin{aligned}
& \nu_{n}=n^{2} \pi^{2} / d^{2} \\
& a_{1}=J_{s} k /\left(K_{s} k_{1} d G \rho_{1} g W\right) \\
& a_{2}=-\left(d^{2} / 6\right) a_{1} \\
& a_{3}=\left(2 \pi^{2} / d^{2}\right) a_{1} \\
& h_{n}=(-1)^{n} d^{4} / n^{2} \pi^{4}
\end{aligned}
$$


which corresponds to using equation 14 for $\Gamma(t)$. This latter case, which applies when the sedimentation rate is small, considerably simplifies the computation of $l(t)$. Also note that the terms in the infinite sum in (15) become small rapidly as $t$ increases.

The results we have so far presented permit us to write a general differential equation for an arbitrary sedimentation law for the solution in this regime. From equations 1,9 , and 12 and the definition of $l(t)$, we obtain the relation between water depth (or elevation) $w(t)$ and the sediment thickness $s(t)$

$q_{1} \frac{d w}{d t}=-\omega \kappa_{1} q_{1} w(t)-q_{2} s(t)+q_{3} \frac{d s(t)}{d t}+q_{4} w_{0}$

where

$$
+q_{5} \sum_{n=1}^{\infty}(-1)^{n} n^{2} e^{-\left(\kappa_{1} n^{3} \pi^{3} / d^{3}\right) l} \int_{0}^{l} e^{\left(\kappa_{1} n^{3} \pi^{3} / d^{n}\right) z} s(z) d z
$$

$$
\begin{aligned}
q_{1} & \equiv \frac{Q_{w}}{\left(1-\rho_{1} / \rho_{2}\right)} \\
q_{2} & \equiv \frac{\varphi \rho_{1} \kappa_{1} W Q_{s}}{\left(1-\rho_{1} / \rho_{2}\right) \rho_{s}} \\
q_{3} & \equiv \kappa_{1}{ }^{2} \epsilon-\frac{\left(1-\rho_{s} / \rho_{r}\right)}{\left(1-\rho_{1} / \rho_{2}\right)} \\
q_{4} & \equiv \frac{\omega \kappa_{1} Q_{w_{o}}}{1-\rho_{1} / \rho_{2}} \\
q_{s} & \equiv \frac{2 \pi^{2} \kappa_{1}{ }^{2} c_{1} J_{s}}{d^{3} H K_{s}}
\end{aligned}
$$

Equation 18 can be integrated once a sedimentation law $s(t)=S(w(t), t, \ldots)$ is specified.

As mentioned above, the regime of long-term blanketed response is probably the most important period. In so far as the precise details of motion during the short term period are not of critical interest, we can use equation 18 as a reasonable approximation to describe the behavior of the basin over all time domains. This will permit a rather good analysis of the general development of the basin and will demonstrate the basic dependence of the model on the choice of physical parameters.

\section{PERIODIC SOLUTIONS}

One of the critical questions pertaining to a phase-change model is whether or not the processes of deposition and uplift can be repeated. The investigations by MacDonald and Ness [1960] in no way answered this question, since they did not consider isostasy or extend their solution to a long enough time. Errors in their analysis have been discussed by Wetherill [1961]. More than one cycle of sedimentation and erosion was found by van de Lindt [1967], who considered a model by MacDonald and Ness with isostasy. However, it is not possible to assess the importance of this owing to possible errors in his solution, which have been discussed by O'Connell [1968]. 
Joyner's [1967] solution showed more than one cycle of sedimentation and erosion. However, the coarse net size -used in his numerical solution makes it difficult to assess the reality of this cyclical phenomenon. We shall show that cyclical processes do in fact occur in such a model, and that they are the result of a time lag between the effects of surface pressure changes and surface thermal effects on the phase boundary.

The existence of such periodic or cyclic solutions is of fundamental importance in that no external mechanism is required to reinitiate the formation of a basin other than the self-sustaining process of sedimentation and erosion.

The general differential equation that we wish to investigate for oscillatory solutions is equation 5 . As mentioned before, this is a nonlinear equation because $Q_{20}$ is a function of $w$. In the ensuing discussion we shall linearize the equation by neglecting the change in $Q_{10}$. Presumably, the solutions obtained with either value of $Q_{w}$ (for $w>0$ and $w<0$ ) should give approximate bounds to the type of behavior to be expected for the solution of the nonlinear equation.

The regime of greatest interest is that of long-term behavior. The corresponding differential equation is equation 18 , given in the preceding section. The solution can be obtained by direct integration, given the initial conditions and the sedimentation and erosion law. A simple law that is geologically plausible is

$$
d s / d t=\beta w
$$

where $\beta$ is a constant. This states that the rate of sedimentation is proportional to water depth and that erosion is proportional to the elevation of the surface above sea level. The limitations of this assumed law will be discussed later.

Assuming this law, we investigate the possibility of solutions for which $w(t)$ is periodic. Taking

$$
w(t)=C_{1} e^{i \gamma t}
$$

we obtain immediately

$$
s(t)=\frac{\beta}{i \gamma} C_{1} e^{i \gamma t}+\langle s\rangle
$$

where $\langle s\rangle$ is the mean sediment thickness about which the sediment thickness varies sinusoidally.

Substituting this into equation 18 , we obtain as a constant of the motion

$$
Q_{w_{v}} w_{0}=Q_{a}\langle s\rangle+\left(1-\frac{\rho_{1}}{\rho_{2}}\right) \frac{J_{s}\langle s\rangle}{K_{a} G \rho_{1} g W}
$$

This equation relates the mean sediment thickness over a time of several oscillations to the initial depth of the basin and to physical parameters that are deterministic of the mechanism; this may be of considerable utility in relating these parameters to $\langle s\rangle$, which may be determinable from the geologic record.

We also obtain the characteristic equation

$$
\lambda_{1} \gamma^{2}=\lambda_{2} i \gamma+\lambda_{3}+\lambda_{4} \sum_{n=1}^{\infty} \frac{(-1)^{n} n^{2}}{\kappa_{1} \nu_{n}+i \gamma}\left[1-e^{-\left(\kappa_{1} \nu_{n}+i \gamma\right) \iota}\right]
$$


where $\lambda_{1}, \lambda_{2}, \lambda_{3}$, and $\lambda_{4}$ are constants, $v_{n}=n^{2} \pi^{2} / d^{2}$, as in equation 17 , and $i=(-1)^{1 / 2}$.

For long times such that transients have decayed, and using

$$
\sum_{n=1}^{\infty} \frac{(-1)^{n} n^{2}}{n^{2}+\zeta}=\frac{-\pi \sqrt{\zeta}}{2 \sinh (\pi \sqrt{\zeta})}
$$

the characteristic equation becomes

$$
A_{1} \sigma^{2}-A_{2} i \sigma-A_{3}=\frac{(i \sigma)^{1 / 2}}{2 \sinh (i \sigma)^{1 / 2}}
$$

in terms of the dimensionless frequency

$$
\sigma \equiv u+i v \equiv \frac{d^{2}}{\kappa_{1}} \gamma
$$

where $u$ and $v$ are real. The constants in this are:

$$
\begin{aligned}
A_{1} & \equiv \frac{Q_{w} H K_{\mathrm{s}} K_{1}}{\left(1-\frac{\rho_{1}}{\rho_{2}}\right) 2 c_{1} J_{\mathrm{s}} \beta d^{3}} \\
A_{2} & \equiv \frac{G g \rho_{1} K_{s}}{2 J_{\mathrm{s}}}\left[\frac{\kappa_{1} W Q_{w}}{d^{2} \beta\left[1-\left(\rho_{1} / \rho_{2}\right)\right]}-\frac{\rho_{s}}{\rho_{1}}\left(\frac{1}{2}+\frac{c_{2}}{c_{1}} \frac{d^{\prime}}{d}\right)\right]+\frac{H K_{s}\left[1-\left(\rho_{s} / \rho_{c}\right)\right]}{2 d c_{1} J_{\mathrm{s}}\left[1-\left(\rho_{1} / \rho_{2}\right)\right]} \\
A_{\mathrm{a}} & \equiv \frac{G g \rho_{1} K_{\mathrm{s}} W Q_{s}}{2 J_{\mathrm{s}}\left[1-\left(\rho_{1} / \rho_{2}\right)\right]}
\end{aligned}
$$

This equation in the complex frequency $\sigma$ can be written in terms of the real variables $u$ and $v$ :

where

$$
\begin{gathered}
A_{1}\left(u^{2}-v^{2}\right)+A_{2} v-A_{3}=\frac{z \sinh z \cos y+y \cosh z \sin y}{2\left[\sinh ^{2} z \cos ^{2} y+\cosh ^{2} z \sin ^{2} y\right]} \\
2 A_{1} u v-A_{2} u=\frac{y \sinh z \cos y-z \cosh z \sin y}{2\left[\sinh ^{2} z \cos ^{2} y+\cosh ^{2} z \sin ^{2} y\right]}
\end{gathered}
$$

$$
\begin{array}{lll}
z \equiv z(u, v)=r \cos \theta & y \equiv y(u, v)=r \sin \theta \\
r \equiv\left(u^{2}+v^{2}\right)^{1 / 4} & \theta \equiv \frac{1}{2} \tan ^{-1}\left(-\frac{u}{v}\right) \\
0 \leq \theta \leq \frac{\pi}{4} \quad v \leq 0 & \frac{\pi}{4} \leq \theta \leq \frac{\pi}{2} \quad v \geq 0 \\
u \geq 0 & &
\end{array}
$$

When the root $\sigma$ is real (i.e., $v=0$ ) there will be a purely oscillatory solution with period $2 \pi d^{2} / u_{\kappa_{1}}$. In general, a real root for $\sigma$ will not exist, and values of $u$ and $v$ (the real and imaginary parts of $\sigma$ ) must be found by solving equations 22 and 23 simultaneously; the solution for the water depth will be

$$
w(t)=C_{1} \exp \left(i u \frac{\kappa_{1}}{d^{2}} t-v \frac{\kappa_{1}}{d^{2}} t\right)
$$

If the value of $v$ is positive, the solution will exhibit damped sinusoidal behavior; 
if $v$ is negative, the solution will be explosive in that the amplitude of the oscillations will grow exponentially with time.

The right-hand sides of equations 22 and 23 are shown in Figures 4 and 5 . We may explicitly examine the conditions for the existence of roots for the two special cases $u=0$ or $v=0$.

1. Undamped oscillations, i.e., $v=0$. In this case, the left-hand side of 22 is

$$
A_{1} u^{2}-A_{3}
$$

which is the parabola, concave upward, that intersects the vertical axis at $-A_{3}$.

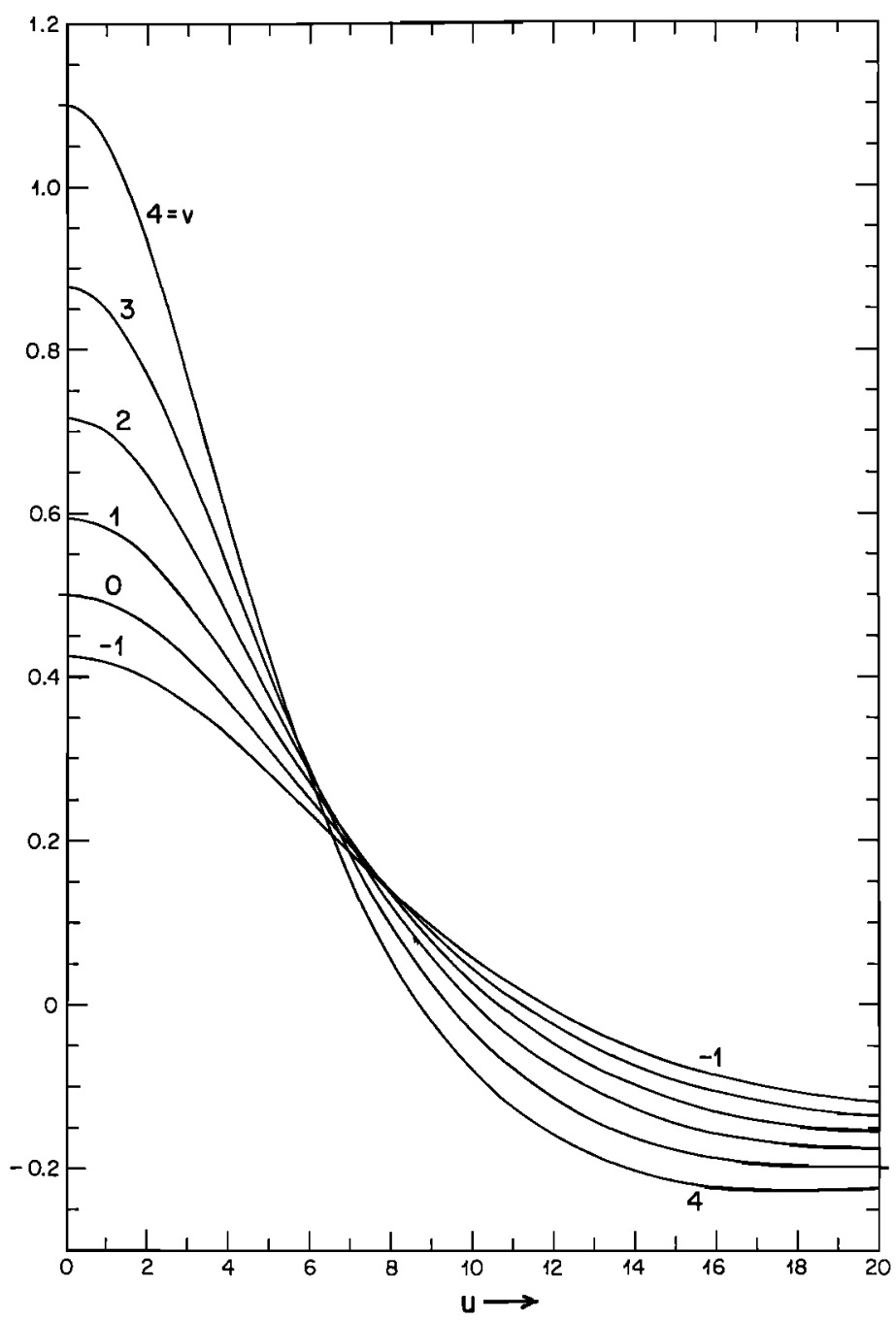

Fig. 4. Right-hand side of equation 22, which is function for determining frequency of oscillatory solution for model with deposition or erosion rate proportional to water depth or elevation. Frequency equals $(u+i v)_{\kappa_{1}} / d^{2}$, and $u$ and $v$ satisfy $A_{1}\left(u^{2}-v^{2}\right)+$ $A_{\mathbf{2}} v-A_{\mathbf{s}}=$ function illustrated, as well as satisfy the equation of Figure 5. 
Thus a solution to equation 22 with $v=0$ will exist for $A_{3}-\geq 1 / 2$ (cf. Figure 4).

However, equation 23 must be satisfied at the same time. The left-hand side of (23) becomes $-A_{2} u$, which is a straight line of slope $-A_{2}$, which passes through the origin. Thus (23) can have a root for $u$, with $v=0$, only if $A_{2} \leq$ $+1 / 12$. However, this root will not, in general, be the same as the root for (22). A solution will occur only for special cases such that (22) and (23) can be satisfied simultaneously with $v=0$. We may conclude, then, that an undamped oscillatory solution will in general occur only for special cases.

2. Nonoscillatory solution, i.e., $u=0$. In this case (22) becomes

$$
-A_{1} v^{2}+A_{2} v-A_{3}=-A_{1}\left(v-\frac{A_{2}}{2 A_{1}}\right)^{2}-\left(A_{3}+\frac{A_{2}{ }^{2}}{4 A_{1}}\right)=\frac{(v)^{1 / 2}}{2 \sin (v)^{1 / 2}}
$$

If $v<0$, the right-hand side becomes

$$
\frac{(-v)^{1 / 2}}{2 \sinh (-v)^{1 / 2}}
$$

where $-v$ is positive. This function is shown in Figure 6 .

The left-hand side of (24) is a parabola, convex upward, with a maximum of $-\left(A_{3}+A_{2}{ }^{2} / 2 A_{1}\right)$ at $v=A_{2} / 2 A_{1}$. Examination of Figure 6 indicates that there will always be a root of (24) near $v=\pi^{2}$, where the parabola will intercept the branch coming from $-\infty$. This root does not interest us, however, since we have assumed that the time was such that the exponential terms in (19) may be neglected, viz., exp $\left(\kappa_{1} \pi^{2} t / d^{2}\right) \ll 1$. A solution with $v=\pi^{2}$ would be proportional to

$$
\exp -\left(\kappa_{1} \pi^{2} t / d^{2}\right)
$$

and would thus be small by this time.

There will be a negative root of (24) if $-A_{3}>1 / 2$, as well as a positive

Fig. 5. Right-hand side of equation 23 analogous to Figure 4 . Roots $u$ and $v$ must satisfy $2 A_{1} u v$ $-A_{2} u=$ function illustrated, as well as satisfy the equation of

Figure 4.

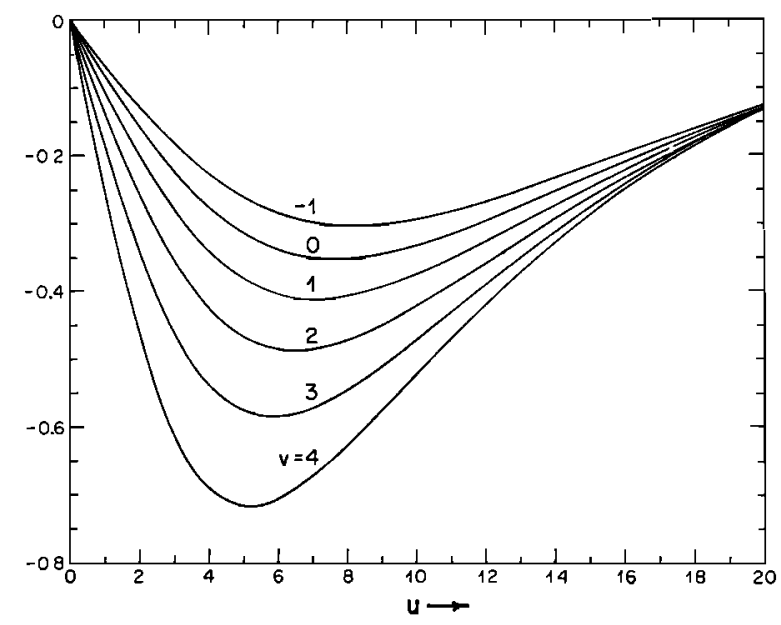




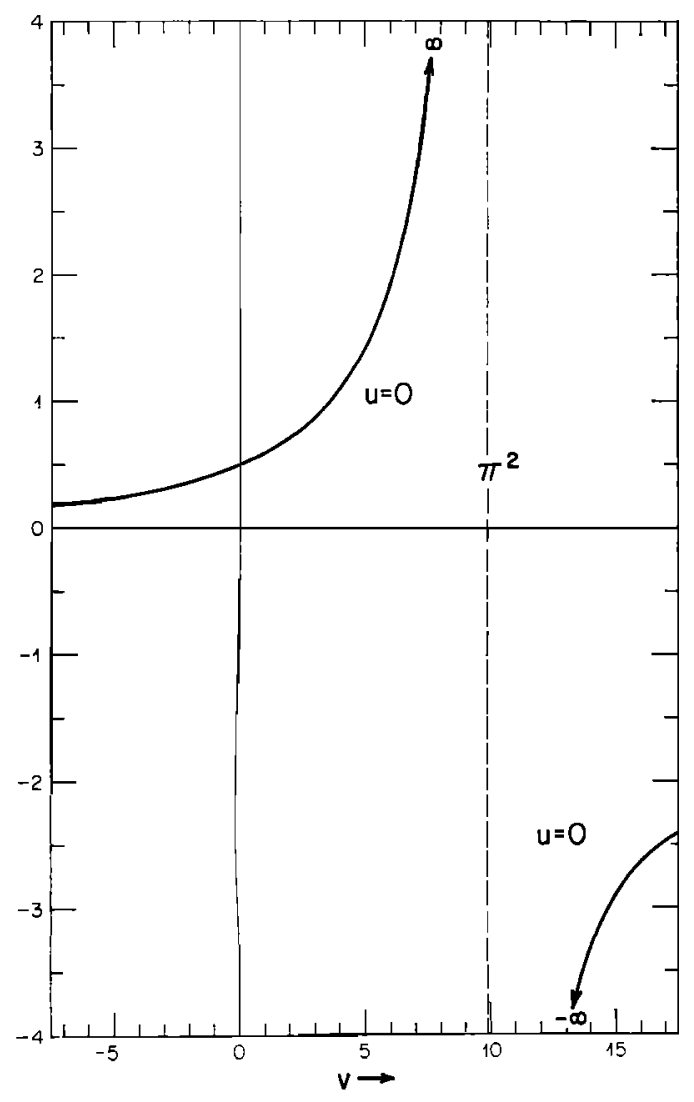

Fig. 6. Function given in Figure 4 plotted as a function of $v$ with $u=0$. If $v>0$, the solution decays exponentially; if $v<0$, the solution grows exponentially. The root $v$ (with $u=0$ ) must satisfy equation 24 , the right-hand side of which is the function shown. Roots from the branch that approaches $-\infty$ at $v=\pi^{2}$ decay so rapidly as to be unimportant. A growing solution with $v<0$ will exist if $-A_{s}>\frac{1}{2}$.

root. The negative root, however, will give rise to a solution that will grow exponentially, and hence will dominate. Two positive roots could exist only if $-A_{3}<1 / 2, A_{2}>1 / 2$, and $-\left(A_{3}+A_{2}{ }^{2} / 4 A_{1}\right)>1 / 2$, which in turn requires $A_{1}<0$. However, $A_{1}>0$ if $Q_{D}>0$; thus two positive roots are not possible unless $Q_{v 0}<0$.

For $u=0,(23)$ is always satisfied identically; thus the above-mentioned roots of (24) are solutions.

From these two limiting cases, then, we conclude (1) that a purely oscillatory solution is a special case and will not occur in general, and (2) if $A_{3}<-1 / 2$, a solution will exist that grows exponentially. From (21), the condition for an exponentially growing, nonoscillatory solution is

$$
Q_{s}<\frac{-J_{s}}{K_{s} G g \rho_{1} W}\left(1-\frac{\rho_{1}}{\rho_{2}}\right)
$$

In general, roots $u$ and $v$ of (22) and (23) will have to be found by solving the equations, which must be done by trial and error or some other such process.

\section{MODEL BASIN CALCULATIONS}

The equations that permit the solution of the history of a sedimentary basin underlain by a phase boundary have been presented in the previous section. 
We now present examples of how these equations can be applied to specific models. We quantitatively consider the histories of two models of depositional basins. The models, $A$ and $B$, described in Table 1 , are essentially similar to models 2 and $5 c$ of Joyner [1967], who solved the field equations directly by numerical methods.

The first model (A) is a sedimentary basin initially $1.5 \mathrm{~km}$ deep being filled at a rate of 200 meters per $10^{6}$ years. If we calculate $Q_{s}$ (equation $1 b$ ), we see that the model is stable. The maximum sediment thickness the basin can accommodate is $10.9 \mathrm{~km}$ (equation 2), which would take 55 million years. From the criteria we have given and the values given in Table 1, short-term behavior will end by 4.1 million years, thermal blanketing will dominate by 14 million years (equation 11), and long-term behavior will apply after 22 million years.

The configuration of the basin with time is shown in Figure 7, in which

TABLE 1. Model Parameters

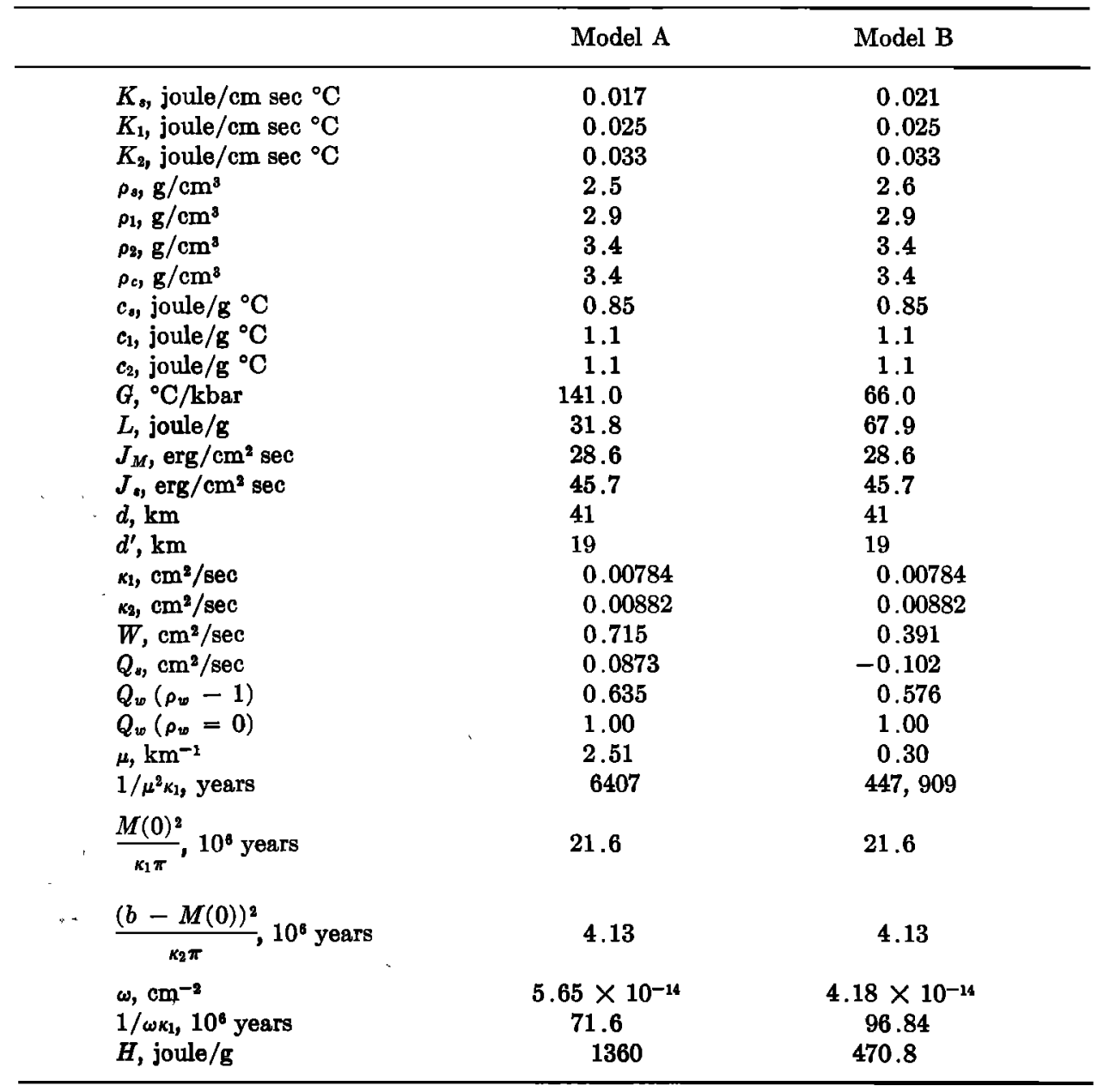




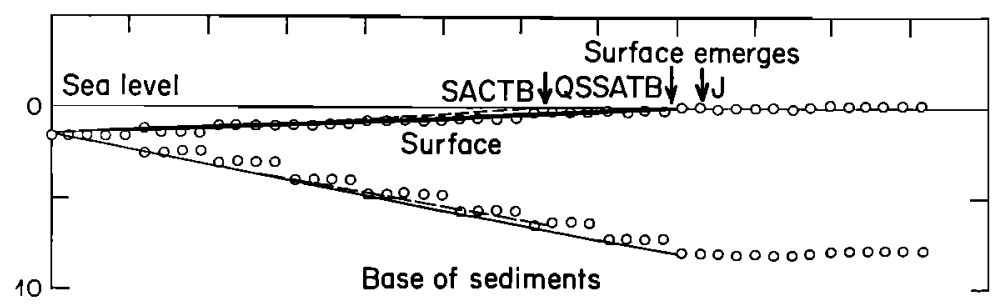

Depth (km)

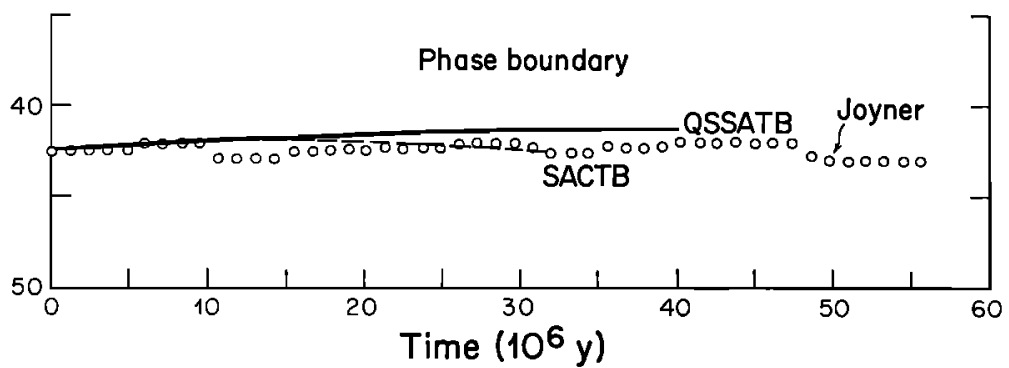

Fig. 7. Results for Model A. The long-term solution (QSSATB) is shown (solid line) as are the short-term solution (SACTB - dashed line) and Joyner's numerical solution (dotted line). The time at which the surface emerged above sea level is indicated for each solution. The numerical solution probably overestimates the time of emergence (see text). Solution QSSATB is the proper solution; the results for solution SACTB were included to show that it is not in gross error even though it is beyond its regime of applicability. This comparison shows that these simple analytic approximations for the temperature field are quite adequate and permit a direct calculation of the evolution of the basin without numerical solution of the field equations.

Joyner's numerical solution (dotted line) is also shown. Solution SACTB is obtained by integrating equation 6 using equation 13 and is appropriate for short-term motion, including the effect of thermal blanketing. Solution QSSATB is that given by equation 15 and is appropriate for long-term blanketing behavior. Note that the two solutions are virtually identical during short-term response (4 million years), and that the use of the long-term solution during this initial period would introduce no significant error. Thus it is unnecessary to start the long-term solution at the time of transition from short-term to long-term behavior with the value of $l(t)$ at this time given by the short-term solution (this is discussed more fully in $O^{\prime}$ Connell $[1969$, p. 134] ).

The basin becomes full (i.e., $w=0$ ) during the period of long-term response; the time when the basin fills $t_{f}$ is obtained from equation 15 by using equation 12 for the lag and yields a time of filling the basin of 40 million years, which is indicated by the vertical arrow labelled QSSATB in Figure 7. (Also shown for comparison are the emergence times for the solution SACTB and Joyner's numerical solution $J$.) The sediment thickness when the basin fills is $s\left(t_{f}\right)=\mathbf{7 . 9}$ $\mathrm{km}$, which is $73 \%$ of the maximum thickness of $10.9 \mathrm{~km}$ (equation 4 ), which would take 55 million years to be deposited.

We now investigate another type of model (model B), which is a sedi- 
mentary basin initially 500 meters deep being filled at a rate of 30 meters per million years. We calculate $Q_{s}=-0.102$, hence the model is unstable. This is due to the similarity of the Clapeyron slope and the temperature gradient at the phase boundary, which results in a small value of $W=\mathbf{0 . 3 9 1}$ (Table 1, equation $1 c$ ). This in turn allows the negative term in $Q_{s}$ (equation $1 b$ ) to dominate, making $Q_{s}$ negative.

Because the model is unstable, a simple static analysis with $l=0$ will not work; e.g., we cannot calculate a maximum sediment thickness from equation 4 . We must therefore investigate the time dependence of the model, since the dynamic effects, the time dependence of the lag, and the effect of thermal blanketing on this are of paramount importance and will determine the dynamic stability of the model and the amount of sediments the basin can accommodate before filling up.

To obtain the long-term response for model $\mathrm{B}$, we use equations 15 and 16 for the lag. These equations apply only until the basin fills and erosion starts. After this time, equation 9 must be integrated by using equation 12 for the effect of thermal blanketing. The result is shown in Figure 8. (Only the longterm solution from time $t=0$ is shown, since it did not deviate significantly from the short-term solution during the 4-million-year period of short-term response.) During the first 50-60 million years of sedimentation, the water depth remains essentially constant, even though $1.5 \mathrm{~km}$ of sediments are deposited in a basin only 500 meters deep. This is a consequence of the dynamic instability of the model (cf. Figure 2). Only after 100 million years does the basin finally become full and the upper surface of the sediments become exposed to erosion. The erosion rate is 15 meters per million years, which is $1 / 2$ the sedimentation rate.

The surface is maintained above sea level until all the sediments that were deposited are eroded away, which takes 200 million years. Even basement rock is exposed to erosion for 30 million years before the surface again subsides below sea level, at which time another cycle of sedimentation, uplift and erosion begins. During the period shown in Figure 8, the motion of the phase boundary relative to sea level is restricted to a $3-\mathrm{km}$ interval, although it moves $5-1 / 2 \mathrm{~km}$ relative to the material in the upper crust.

Figure 8 clearly shows that more than one cycle of sedimentation and erosion can take place, once the process is started. Figure 9 shows the same solution as in Figure 8, continued for a longer time $(1,000$ million years versus 450). The similarity of the last three cycles indicates that the solution is periodic and undamped, once the initial transients from the first cycle have disappeared. During each cycle of 234 million years, $2.21 \mathrm{~km}$ of sediments are deposited, whereas the water depth never exceeds 120 meters. The resulting ratio of sediment thickness to water depth is 18.4; this exceeds, by far, the value of 6 for the first cycle and is a striking illustration of the amount of subsidence that a phase-change mechanism can account for.

\section{COMPARISON WITH NUMERICAL SOLUTIONS}

It is of interest to compare our analytic solutions with the numerical solutions obtained by Joyner [1967] for models A and B, which are essentially 


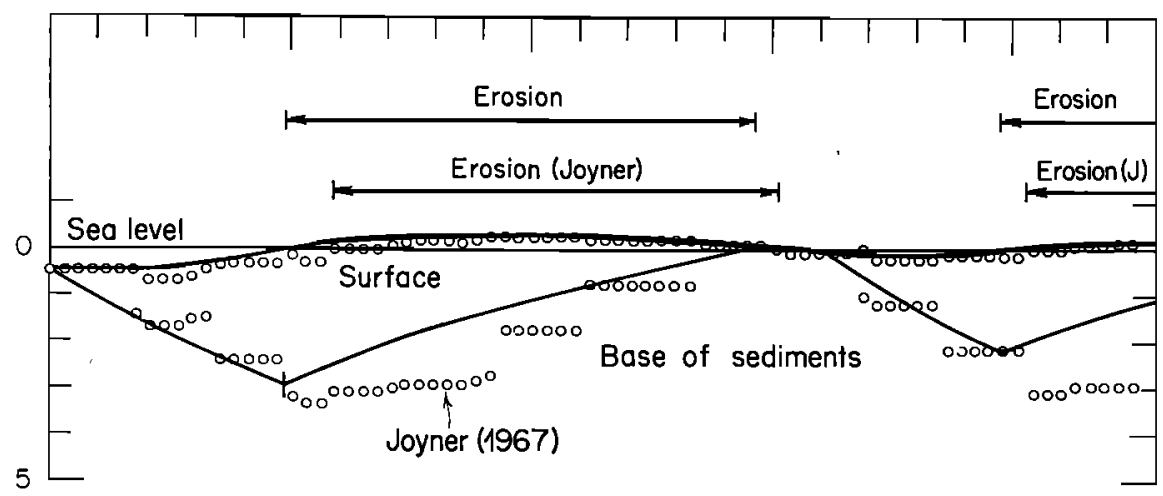

Depth (km)

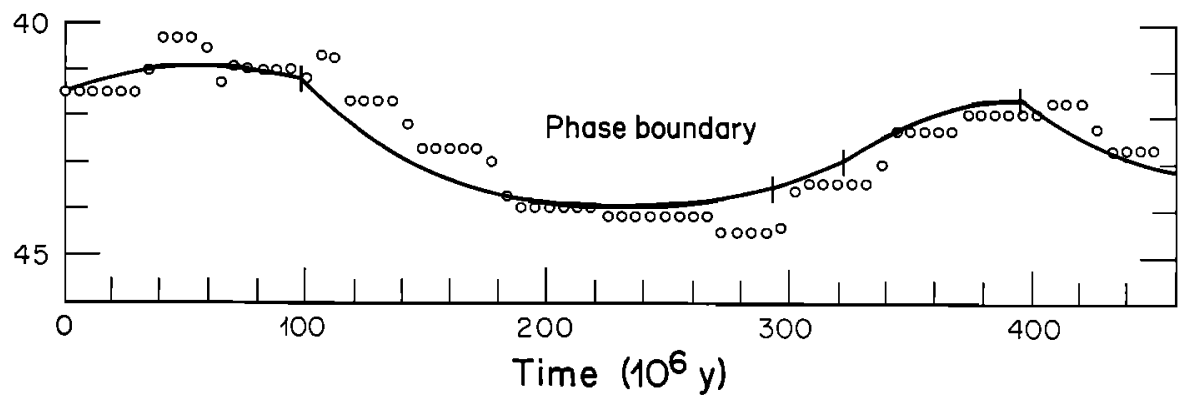

Fig. 8. Results for Model B. During two cycles of deposition and uplift and erosion, the long-time solution from equation 15 agrees excellently with Joyner's numerical solution. The differences that do exist are due to the different erosion laws of the two solutions (see text) and the discontinuous sedimentation in the numerical solution. The instability of the model is manifested by the failure of the water depth to decrease during the first $\mathbf{5 0}$ million years of deposition.

identical to Joyner's models $2 a$ and $5 c$. The numerical solutions are shown as dotted lines in Figures 7 and 8 . The agreement between the analytic and the numerical solutions is very good. The irregularities in the numerical solution arise partly because in the numerical solution sediments were deposited in increments of $1 \mathrm{~km}$, rather than continuously; e.g., in Figure 7, $1 \mathrm{~km}$ of sediments was placed instantaneously on the surface every 5 million years. This mode of deposition could easily cause the time of emergence of the surface (indicated by the vertical arrow labeled $J$ ) to be in error by several million years. The effect of discontinuous deposition is more apparent in Figure 8. Deposition of a slab of sediments causes the phase boundary to move in a steplike fashion, whereas the analytic solution appears like a reasonable smoothed version of the numerical solution during the first 100 million years. We note that the dynamic instability of model B is evidenced in the numerical solution, since the water depth increases when the first slab of sediments is deposited. The importance of this was not mentioned by Joyner. 
The difference between the numerical and analytic solution during the period of erosion in Figure 8 is due to different erosion laws for the two models. Joyner's erosion rate was proportional to elevation; our erosion rate was constant. With this taken into account, the agreement between the two solutions is very good, which suggests that the exact form of the erosion law may not be of paramount importance.

In general we conclude that there is essential agreement between the numerical solutions of Joyner [1967] and our analytic solutions of the same models. In fact, it would require a numerical solution considerably more refined than Joyner's to reveal any shortcomings of the approximate analytic solution we have developed. In addition, the repeated cycles of sedimentation and erosion shown in Figure 9 demonstrate that the cycles shown in Joyner's solution were not merely consequences of the numerical technique used but are implicit in the model itself.

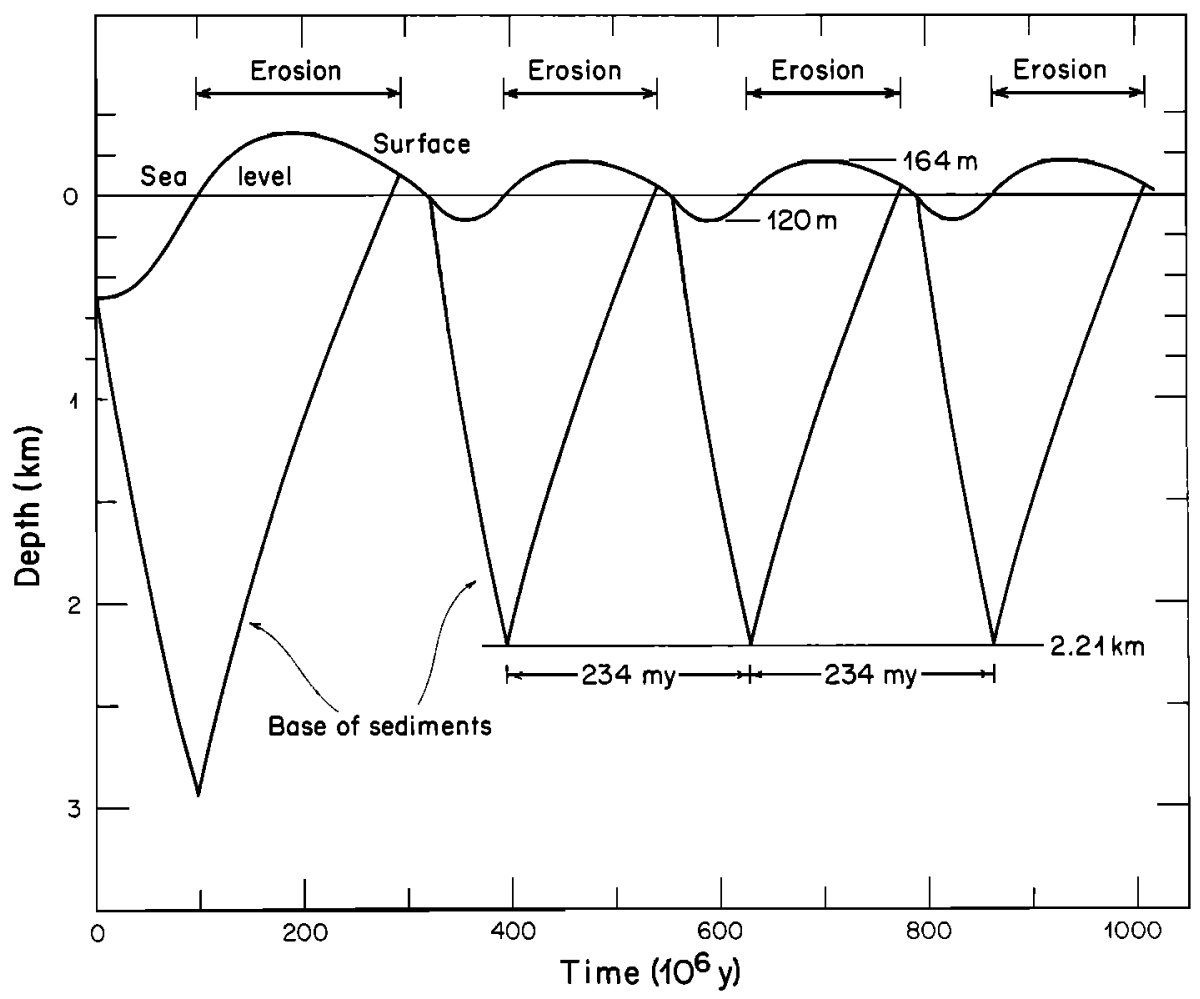

Fig. 9. Analytic solution of Model B of Figure 8 extended to $10^{9}$ years, showing the existence of undamped oscillations after the first cycle of deposition and erosion. During the 80-million-year deposition phase of each cycle, $2.21 \mathrm{~km}$ of sediments are deposited in water that never exceeds 120 meters in depth. During the erosion of these $2.21 \mathrm{~km}$ of sediments, a surface elevation of up to 164 meters is maintained. In this solution, deposition occurs at a constant rate when the surface is below sea level, and erosion occurs at a constant rate when the surface is above sea level. 


\section{PERIODIC SOLUTIONS}

Model B can also be investigated for the existence of sinusoidal solutions of the form

$$
w(t)=C_{1} e^{i \gamma t} \equiv C_{1} \exp \left(i u \frac{\kappa_{1}}{d^{2}} t-v \frac{\kappa_{1}}{d^{2}} t\right)
$$

The sedimentation rate is proportional to water depth, with the ratio $\beta$ ranging from 0.1 to 0.5 per million years. We see from equation 19 that initial transients will decay by a factor of $e$ in 7 million years; our analysis will therefore be applicable for processes that take a time that is considerably longer than this time, as will be the case. The constants $A_{\imath}$ in the characteristic equation 20 are given in terms of the model parameters by equation 21. Equations 22 and 23 can be solved by an iterative procedure.

The dimensionless frequency roots $u$ and $v$ are given in Table 2, along with the period of the oscillations (time for one cycle of deposition and erosion) and the $e$-folding time of the growth or decay of the oscillations (time for solution to grow or decay by a factor of $e$ ). The roots are given for several sedimentation rate constants $\beta$, and for two different values of $Q_{w}$ corresponding to cases with water either absent or present at all times. Because the asymmetric case, with water present during deposition but absent during erosion, is nonlinear, it is not amenable to the same analysis for roots that is possible for the linear cases we consider (cf. equation $5 \mathrm{ff}$.). Nevertheless, the behavior of the linear cases should bracket the behavior of the nonlinear case. If the solution of the nonlinear case is desired, it can always be obtained by integrating equation 18 directly, as was done for the results shown in Figures 8 and 9.

The periods of oscillation in Table 2 range from 132 to 351 million years. Since the amplitudes of the oscillations are not determined, the absolute sedimentation rates are not known. However, for an average water depth of 100 meters, the rates corresponding to our values of $\beta(0.1-0.5)^{-1}$ million years would be $10-50$ meters $/ 10^{6}$ years. The sediment thickness will oscillate around the mean value $\langle s\rangle=2.8 \mathrm{~km}$. If more than $2\langle s\rangle=5.6 \mathrm{~km}$ are eroded in any one cycle, the basement rock will be exposed to erosion. At an erosion rate of 50 meters $/ 10^{6}$ years this would require 112 million years, corresponding to a period of 224 million years. This period is comparable to those in Table 2 ; thus the erosion of all the sediments and the exposure of basement rock is clearly possible for this model.

The number of exponentially growing solutions in Table 2 is rather striking. The most rapidly growing of these will increase in amplitude by a factor of $e$ in 111 million years, which is less than one period; in less than two periods the oscillations will grow in amplitude by a factor of ten. Even if the basin was initially very shallow, the rapid growth would result in the deposition and erosion of considerable thicknesses of sediments, and eventually basement rock.

The values for the period and growth or decay rates in Table 2 are consistent with the solution shown in Figure 8, although the sedimentation laws are quite different. The solution in Figure 9 has an average sedimentation/ erosion rate of $\sim(1 / 2)(30+15)=22.5$ meters $/ 10^{6}$ years and an average 


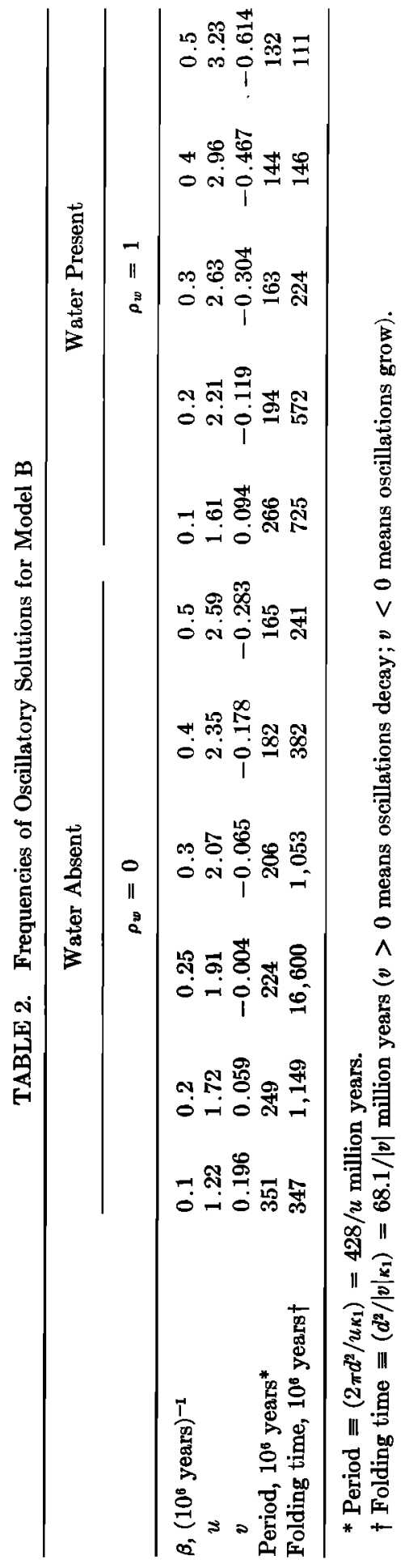


water depth/elevation of $\sim 100$ meters. This corresponds most nearly with the solutions in Table 2 with $\beta \sim 0.2$, which have periods of $\sim 225$ million years and a small growth or decay constant, so that the solutions are nearly constant in amplitude.

The solutions with water present $\left(\rho_{v}=1\right)$ are less stable than those with water absent. This may seem inconsistent, since the deposition of sediments in water would reduce the effective density of the sediments, owing to the buoyant effect of the water. Referring to Figure 9, however, we see that as sediments are deposited the water depth is increasing, thereby increasing the load on the surface and making the phase boundary more responsive to the surface load, which promotes instability.

For any oscillatory solution, the ratio of sediment thickness deposited to the maximum water depth can be considerable, since the period of the oscillation, and hence the time available for deposition, is independent of the water depth. Thus if the period is 200 million years, and the average water depth is 100 meters, then for $\beta=0.3$ (million years) ${ }^{-1}, 3 \mathrm{~km}$ of sediments will be deposited, and the maximum water depth will be 157 meters. The ratio of sediment thickness to water depth will be 19.1. In fact, this ratio is independent of the water depth, and is approximately equal to $\beta T / \pi$ where $T$ is the period of the oscillation. The value of 19.1 obtained above can be contrasted with the value of 3.0 for this ratio if there were no phase change but only isostasy.

The existence of oscillations as solutions of an equation arising from heat diffusion may seem anomalous. The oscillatory solutions, and even the presence of uplift after deposition, associated with phase-change models are cases of a system 'overshooting' when it moves to equilibrium. This arises for phasechange models because, although the pressure change due to the sediments is transmitted to the phase boundary essentially instantaneously, the thermal effect of the sediments is only transmitted with a velocity characterized by $\kappa_{1} / d$, which is of the order of $10^{-14} \mathrm{~km} / \mathrm{sec}$. The resultant phase lag between the two effects at the phase boundary causes the phase boundary to move toward a 'false' equilibrium position, false because it is determined by the thermal effect from a previous configuration of sediments and the pressure effect from the present configuration. Anything that tends to increase or speed up the thermal effect at the phase boundary will tend to reduce the phase lag between pressure and temperature effects and hence the period of any oscillations. Thus a more rapid sedimentation rate, by causing a more rapid change of surface thermal conditions, tends to reduce the period of oscillation, as is seen in Table 2. A lower value of thermal conductivity for the sediments, a shallower phase boundary, a higher surface-heat flux, or a higher value of $\kappa_{1}$ would have the same effect. In this manner, the effect of most parameters can be predicted and understood.

\section{SUMMARY}

The results of the previous section demonstrate the accuracy and simplicity of the approximate analytic solution for the motion of a phase boundary. The simplicity is noteworthy, especially in comparison with the complete set of 
partial differential equations describing the problem (cf. OW), which require rather complex and time-consuming techniques even for numerical solution. By using an approximate expression for the temperature field, the problem has been reduced to the solution of an ordinary differential equation, which can be directly integrated in many cases.

The general technique used lends itself to other problems of this type and deserves outlining.

The first step is to isolate the problem of the moving phase boundary from the other processes in the model. This was done by defining the lag of the phase boundary $t$, with which all the transient effects of the moving phase boundary are associated. This reduces the problem to that of solving for the response of the phase boundary, which was presented in OW. The subsequent steps that lead to the solution are:

1. Subtracting the initial steady-state temperature distribution; reducing the problem to solving for the change in temperature (defined as the perturbation temperature.)

2. Finding the solution for short times by representing the temperature by a modification of the solution for freezing at a constant temperature.

3. Finding a long-time solution by using the steady-state temperature distribution that applies for each instantaneous position of the phase boundary.

4. Including the effects of thermal blanketing by superimposing the solutions for blanketing and the motion of the phase boundary.

Although this paper treats one fairly specific model of an upper-mantle phase change, the method is flexible and of wide applicability. For example, different boundary conditions are included by modifying the quasi-steady-state temperature distribution in step 3, permitting even time-dependent conditions to be included. Such cases could arise from studying phase changes in a lithospheric slab as it moved over regions of the upper mantle at different temperatures, for example.

The reduction of the problem of a moving phase boundary to the solution of an ordinary differential equation permits many important characteristics of the problem to be immediately assessed. In particular, the dependence of the solution on certain parameters of the model is immediately obvious. This is of considerable importance in a geophysical problem of this type, since the values of many parameters are not known with certainty, and it is important to know to what extent the solution depends on the values chosen for these parameters.

For short-term motion, the primary rate constant is the parameter $\mu$ in equation 6. This is essentially the ratio of the difference between the Clapeyron slope and the geotherm to the latent heat of the phase change. A larger value of $\mu$ would make the phase boundary more responsive, as can be seen from the solution given in equation 8.

For long-term motion, the primary rate parameter is w (see equation 9). This depends mostly on the parameters specifying the depth of the phase boundary and the position at which the lower boundary condition is applied, which is a reflection of the dependence of long-term behavior on the diffusion of 
heat over the entire region. Correspondingly, it can be seen that the value of the latent heat $L$ is relatively unimportant in so far as it appears in combination with other terms that may be considerably larger. Similarly, one can see from equation 14, that thermal blanketing depends primarily on the surface gradient, the rate of loading, and the depth of the phase boundary.

Some of the limitations of our model should be discussed. Our use of a one-dimensional model is clearly a simplification. Yet, for shallow phase boundaries at depths $\sim 50 \mathrm{~km}$, and for areas more than $\sim 100 \mathrm{~km}$ in extent, the one-dimensional approximation is probably quite good. For deeper phase changes or more limited regions, where the lateral fow of heat may be significant, the one-dimensional model exaggerates the effects associated with the phase boundary. Nevertheless, the effects should be qualitatively the same as those predicted but reduced in amplitude somewhat.

The boundary condition applied at the lower boundary has an important effect on the long-term motion of the phase boundary, as has been discussed by OW. We have used a condition of constant flux in the models treated in this paper. The proper boundary condition that should be applied is not obvious. Therefore, the effect of different boundary conditions should be assessed, as was done in OW. In general, a constant temperature boundary condition results in a more rapid motion of the phase boundary during long-term response than does a constant flux condition; moreover, a deeper boundary condition lengthens the time required to reach long-term response.

Our treatment of the motion of the phase boundary has assumed that it is a plane of separation between two phases, and that no mixed-phase region exists. Phase transitions in the earth may be characterized by transition regions of mixed phase that may be several kilometers thick. The effect of a finite mixed-phase region on our solution should be examined.

For very short times, the width of the temperature disturbance near the phase boundary will be narrow (cf. Figure 3). If this width is of the same order or less than the thickness of a transition zone, the assumption of a plane phase boundary will not be very good. For longer times, however, when the width of the temperature disturbance is considerably wider than the transition region, the finite width of the phase boundary should have only a limited effect, since it will be narrow compared to the temperature disturbance. In this case, the assumption that the phase boundary is infinitesimal in width should be good, because the diffusion of latent heat away from the phase boundary will be controlled by the transport of heat over the whole region of the temperature disturbance. Thus, it will not be strongly dependent on details near the phase boundary. Such will be the case during long-term response, so that we may expect that the existence of a mixedphase region should have little effect during this type of behavior.

Our model assumes instantaneous isostatic compensation of changes in the configuration of the model. The time scale of the phase-change mechanism for the type of examples we have considered is tens of millions of years and is thus much longer than the time scale of $\sim 10,000$ years for isostatic readjustment. Thus, our assumption of instantaneous compensation is well founded. A finite rate of isostatic compensation comparable to the relation time of the phase change would make 
the problem more complex but could be included. It would lead to the limit of no isostatic response, which is included in the general solution by letting $\rho_{\sigma} \rightarrow \infty$.

The fact that the model requires compensation only of changes in the configuration of the model means that isostatic anomalies may still exist, if due to other causes. A basin corresponding to our model could thus exist in a region with an intrinsic isostatic gravity anomaly. The basin itself would not change the preexisting anomaly as sedimentation uplift and erosion occurred, and the anomaly would not necessarily reflect the presence or history of the basin.

Our model for studying upper-mantle phase changes has assumed that the phase boundary lies above the level at which isostatic compensation takes place. This has led to the operation of both isostasy and the phase change in promoting subsidence. If the phase boundary were beneath the level of isostatic compensation, it would be insulated from long-term pressure changes at the surface by the fluid layer, in which flow takes place to achieve isostatic compensation. Such a deep phase change would play no role then in the processes of subsidence and uplift that we have been considering. Nevertheless, the response of phase changes deep in the mantle to changes in pressure or temperature can be calculated by the same general methods we have used here.

The two sedimentation laws we have investigated, $\dot{s}=$ constant and $\dot{s}=$ $\beta w$ (i.e. proportional to water depth) are clearly idealizations. The fact that both laws yielded periodic solutions and that the periods of the oscillation were roughly the same for models with the same average sedimentation rate indicates that the functional form of the sedimentation law is probably not of prime importance and that_it is the weighted integral of $s(t)$ in_equation_18 that is_important.

The relation of our one-dimensional model to a real basin may perhaps be visualized by considering a region throughout which the elevation, water depth, sediment thickness, and sedimentation rate are all uniform at a given time. This region would move up and down vertically as a plug in response to the processes we have been considering. The parameters that are uniform throughout this area would correspond to the same values averaged over the area of a real basin. For a real basin, the location of a source or sink of sediments attains an importance not present in the one-dimensional model. The same process could take place in two adjacent regions, but out of phase, so that the emergent region would be a source of sediments for an adjacent subsiding basin. Obviously, such considerations are beyond the limitations of a one-dimensional model, yet the results of the model still permit a qualitative discussion of the possibilities.

\section{CONCLUSIONS}

We have presented approximate analytic solutions for the history of an isostatically compensated sedimentary basin (or eroding elevation) underlain by a phase change. These solutions are in closed form and can be evaluated without recourse to high-speed computers. The solutions are accurate, especially in view of the uncertainties of the appropriate parameters that should be used in modeling a basin on the earth. The analytic form of these solutions and their simplicity allows the effect of the parameters of a model to be readily seen. Thus the behav- 
ior of models that are variations of a model that has been solved can be confidently predicted.

In addition, certain characteristics of a model permit a classification of its behavior. Thus, for example, criteria for both long-term and short-term behavior, which are distinct, allow one to consider the behavior of a model in a general sense and permit discussion of models in terms of these general characteristics. There are also criteria that establish generic types of models, such as stable or unstable. Models of one type all share certain important characteristics, which allows one to discuss the behavior and consequences of a sedimentary basin in terms of these general properties, rather than on the basis of individual cases. In essence, the solutions we have presented permit the creation of a vocabulary for discussing the behavior of models without dealing in particulars of any single model.

This vocabulary then allows one to discuss the geological problem in a precise and quantitative manner. Whether or not phase changes actually are an important feature of sedimentary basins, a framework for discussing and investigating the question has been established. With this framework, the roles of phase changes in subsidence and uplift in the earth's history can be investigated in an orderly way. The existence of practicable solutions allows one to predict the consequences of any model; in this way, models can be tested and either discarded or accepted. The conditions on a model for it to be acceptable can be evaluated, and the likelihood of these conditions being fulfilled can be treated as a question apart from the behavior of a model.

\section{APPENDIX}

The inclusion of the effect of thermal blanketing is done as in our previous paper (OW, pp. 379 and 395). We shall linearly superimpose the temperature field due to the movement of the phase boundary and the field from the blanketing effect of the sediments. The heat flux at the phase boundary from both temperature fields is set proportional to the velocity of the phase boundary to obtain a differential equation for the motion of the phase boundary.

Although the problem is nonlinear, the analysis presented in OW has shown that a reasonable solution can be obtained by superposition that satisfies the field equations and the conditions at the moving boundary, while only approximately satisfying the other conditions. In the context of the quasi-steady-state solution used for long-term motion, all the boundary conditions are satisfied, whereas the field equations are only approximately satisfied in so far as $\partial / \partial t \approx 0$. The accuracy of this approximation has been verified by the numerical results in $0 W, O$ and this paper.

To include thermal blanketing, we define $\Theta_{B}(x, t)$ as the change in temperature from the initial steady state that is due to the thermal blanketing effect of the sediments. Initially $\Theta_{B C}(x, 0)=0$ everywhere. As sediments are deposited, the boundary conditions are $\Theta_{B C}\left(x_{s}, t\right)=\left(J_{s} / K_{s}\right) s(t)$ and $\Theta_{B C}(M, t)=0 . J_{\text {, is }}$ ise initial surface heat flux and $K_{\mathrm{a}}$ is the conductivity of the sediments. This defines a change in temperature that increases underneath the sedimentation surface, does not change the temperature at the phase boundary $M$, but does change the heat flux at $x=M$. Assuming 


$$
M(0)-x_{s}(0) \approx M(t)-x_{s}(t)
$$

we can solve the field equation

$$
\frac{\partial \Theta_{B C}}{\partial t}=\frac{\kappa_{1} \partial^{2} \Theta_{B C}}{\partial x^{2}}
$$

in the region $x_{s}(t) \leq x \leq M(t)$ subject to the initial and boundary conditions above.

If we neglect the motion of the phase boundary and sedimentation surface relative to the material between them, the solution follows from Duhamel's theorem, and we obtain the result given in equation 12 for the added gradient at the phase boundary

$$
\left.\frac{\partial \Theta_{B C}}{\partial x}\right|_{x=M^{-}} \equiv \Gamma(t)
$$

For rapid sedimentation the motion of the sedimentation surface relative to the material beneath can be taken into account approximately. Taking $d s / d t$ as a constant average effective sedimentation rate, and assuming as before

$$
\left[M(0)-x_{s}(0)\right] /\left[M(t)-x_{s}(t)\right] \approx 1
$$

the problem is as above with the exception that both the sedimentation surface and phase boundary are moving relative to the material between them at a velocity approximately equal to $d s / d t$. Using a coordinate system $x^{\prime}$ in which the boundaries are fixed and the material is in motion, the field equation becomes

$$
\frac{\partial \Theta}{\partial t}\left(x^{\prime}, t\right)-\frac{d s}{d t} \frac{\partial \Theta}{\partial x^{\prime}}\left(x^{\prime}, t\right)=\kappa_{1} \frac{\partial^{2} \Theta}{\partial x^{\prime 2}}\left(x^{\prime}, t\right)
$$

Using Laplace transforms [cf. Carslaw and Jaeger, 1959, p. 388] we obtain for the added gradient at the phase boundary

$$
\begin{aligned}
\Gamma \equiv-\frac{J_{s}}{K_{\mathrm{o}} \kappa_{1}} \frac{d s}{d t} \exp \left(\frac{d}{\kappa_{1}} \frac{d s}{d t}\right)\left\{\frac{\frac{1}{2} t \frac{d s}{d t}+\frac{\kappa_{1}}{d s / d t}}{\sinh \left(\frac{1}{2} \frac{d}{\kappa_{1}} \frac{d s}{d t}\right)}-d \frac{\cosh \left(\frac{1}{2} \frac{d}{\kappa_{1}} \frac{d s}{d t}\right)}{\sinh ^{2}\left(\frac{1}{2} \frac{d}{\kappa_{1}} \frac{d s}{d t}\right)}\right. \\
\left.-\frac{2 \pi^{2}}{d^{3}} \sum_{n=1}^{\infty} \frac{(-1)^{n} n^{2}}{\left[\frac{\pi^{2} n^{2}}{d^{2}}+\frac{(d s / d t)^{2}}{4 \kappa_{1}^{2}}\right]^{2}} \exp \left[-\left(\frac{n^{2} \pi^{2}}{d^{2}}+\frac{(d s / d t)^{2}}{4 n_{1}^{2}}\right) \kappa_{1} t\right]\right\}
\end{aligned}
$$

In the limit neglecting the movement of the surface and the phase boundary $(U \rightarrow 0)$, we get the result of our previous paper (OW, p. 395) and

$$
\Gamma=-\frac{J_{0}(d s / d t)}{K_{8} \kappa_{1}}\left[\frac{\kappa_{1} t}{d}-\frac{d}{6}-\frac{2 d}{\pi^{2}} \sum_{n=1}^{\infty} \frac{(-1)^{n}}{n^{2}} \exp \left(-\frac{\pi^{2} n^{2}}{d^{2}} \kappa_{1} t\right)\right]
$$

This expression is considerably simpler than (A1), and is sufficiently accurate where

$$
\frac{1}{2} \frac{d}{\kappa_{1} \ltimes} \frac{d s}{d t} \lesssim 0.1
$$


A further simplification comes if the infinite sum on the right of (A1) and (A2) can be neglected. This often is the case by the time that thermal blanketing must be included in the solution for the lag $l(t)$.

Acknowledgments. Contribution 2092 of the Division of Geological and Planetary Sciences, California Institute of Technology, Pasadena, California.

The work was supported by a grant from the National Science Foundation.

\section{REFERENCES}

Carslaw, H. S., and J. C. Jaeger, Conduction of Heat in Solids, 2nd ed., Oxford University Press, New York, 1959.

Joyner, W. B., Basalt-eclogite transition as a cause for subsidence and uplift, J. Geophys. Res., 7\&, 4977-4998, 1967.

MacDonald, G. J. F., and N. F. Ness, Stability of phase transitions within the earth, J. Geophys. Res., 65, 2173-2190, 1960.

O'Connell, R. J., Critique of the paper by W. J. van de Lindt, 'Movement of the Mohorovicic discontinuity under isostatic conditions,' J. Geophys. Res., 73, 6604-6607, 1968.

O'Connell, R. J., Dynamic response of phase boundaries in the earth to surface loading, Ph.D. thesis, Calif. Inst. of Technol., Pasadena, 1969.

O'Connell, R. J., and G. J. Wasserburg, Dynamics of the motion of a phase change boundary to changes in pressure, Rev. Geophys. Space Phys., 6, 329-410, 1967.

van de Lindt, W. J., Movement of the Mohorovicic discontinuity under isostatic conditions, J. Geophys. Res., 7\%, 1289-1297, 1967.

Wetherill, G. W., Steady-state calculations bearing on geological implications of a phasetransition Mohorovicic discontinuity, J. Geophys. Res., 66, 2983-2993, 1961.

(Received June 1, 1971 ; revised November 8, 1971.) 